\title{
Exact Steady State of the Open $X X$-Spin Chain: Entanglement and Transport Properties
}

\author{
F. Benatti, ${ }^{1,2}$ R. Floreanini, ${ }^{2}$ and L. Memarzadeh $\oplus^{3, *}$ \\ ${ }^{1}$ Department of Physics, University of Trieste, Trieste I-34151, Italy \\ ${ }^{2}$ Istituto Nazionale di Fisica Nucleare (INFN), Sezione di Trieste, Trieste I-34151, Italy \\ ${ }^{3}$ Department of Physics, Sharif University of Technology, Tehran 11365-9161, Iran
}

(Received 23 February 2021; revised 18 July 2021; accepted 9 August 2021; published 15 September 2021)

\begin{abstract}
We study the reduced dynamics of open quantum spin chains of arbitrary length $N$ with nearest-neighbor $X X$ interactions, immersed within an external constant magnetic field along the $z$ direction, the end spins of which are weakly coupled to heat baths at different temperatures, via energy-preserving couplings. We find the analytic expression of the unique stationary state of the master equation obtained in the so-called global approach based on the spectralization of the full-chain Hamiltonian. Hinging upon the explicit stationary state, we reveal the presence of sink and source terms in the spin-flow continuity equation and compare their behavior with that of the stationary heat flow. Moreover, we also obtain analytic expressions for the steady-state two-spin reduced density matrices and for their concurrence. We then set up an algorithm suited to compute the stationary bipartite entanglement along the chain and to study its dependence on the Hamiltonian parameters and on the bath temperatures.
\end{abstract}

DOI: 10.1103/PRXQuantum.2.030344

\section{INTRODUCTION}

Transport phenomena in open interacting quantum spin chains have recently received increasing attention as instances of many-body systems driven by intrinsic interspin interactions and coupled to external heat baths at the two ends of the chain. Specific experimental realizations have been reported in scenarios involving ultracold atoms, light-harvesting complexes, and quantum thermodynamics at large [1-21].

In presence of external baths, the reduced dynamics of any open quantum system are obtained by tracing over the degrees of freedom of the baths. When the strength of the system-baths interaction is small compared to the system and bath typical energies, applying the weakcoupling limit techniques yields a dissipative irreversible time evolution that is generated by a master equation in Gorini-Kossakowski-Sudarshan-Lindblad (GKSL) form [22-37].

The derivation of the GKSL master equation requires the diagonalization of the full spin-chain Hamiltonian. Due to the many degrees of freedom of the quantum

*memarzadeh@sharif.edu

Published by the American Physical Society under the terms of the Creative Commons Attribution 4.0 International license. Further distribution of this work must maintain attribution to the author(s) and the published article's title, journal citation, and DOI. spin chain and their mutual interactions, dissipative effects then arise involving all spins in the chain together with environment-induced excitation transfer between different sites (see, e.g., Refs. [38-48]). These give rise to new global effects in transport phenomena that cannot be captured using other, simplified, approaches to the chain open dynamics [49].

In the following, we focus on the study of the stationarytransport and bipartite-entanglement properties of open $X X$ chains with energy-conserving couplings to external baths. We provide an explicit analytic form for the chain stationary state by means of which we obtain analytic expressions for the spin flow, revealing the presence of sink and source terms, and for the heat flow. Remarkably, we are also able to explicitly compute the reduced twospin density matrices resulting from the stationary state and study the corresponding bipartite entanglement along the chain. For the latter task, we develop a suitable algorithmic representation of the stationary state in the spin representation.

The structure of the paper is as follows. In Sec. II, we set the framework for the derivation of the open-chain dynamics and diagonalize the chain Hamiltonian by turning the spin representation into a fermionic one. In Sec. III, we derive the Lindblad operators yielding the dissipative contribution to the GKSL master equation, prove that the latter has a unique stationary state, and explicitly derive its expression in the fermionic representation. Based on this representation, we then discuss the ensuing transport 
properties in terms of spin and heat flows. In Sec. IV, we rewrite the stationary state in the spin representation and show that it provides reduced two-spin density matrices in the so-called $X$ form, which allows for a simple analytic expression of the concurrence. Then, we set up a representation of the stationary state that is best suited for the study of bipartite entanglement and its dependence on the various parameters of the chain and on the temperatures of the baths coupled to it. We conclude by summarizing and discussing the results, while the more technical issues are presented in various appendices.

\section{OPEN $X X$ SPIN CHAIN OF LENGTH $N$}

As mentioned in Sec. I, in the following we address an open quantum chain consisting of $N$ spins at sites $1,2, \ldots, N$, immersed in a constant magnetic field along the $z$ direction, with $X X$ nearest-neighbor interactions among themselves. The ensuing closed chain dynamics are thus generated by the following Hamiltonian:

$$
H=g \sum_{\ell=1}^{N-1}\left(\sigma_{x}^{(\ell)} \sigma_{x}^{(\ell+1)}+\sigma_{y}^{(\ell)} \sigma_{y}^{(\ell+1)}\right)+\Delta \sum_{\ell=1}^{N} \sigma_{z}^{(\ell)},
$$

with free boundary conditions, where $\Delta>0$ is the intensity of the constant transverse magnetic field, $\sigma_{x, y, z}^{(\ell)}$ are the Pauli matrices at site $\ell$, and $g>0$ is the strength of the nearest-neighbor interaction. Throughout the paper, we work in natural units where both Planck and Boltzmann constants are set to $1, \hbar=\kappa_{B}=1$.

The spin chain is then turned into an open many-body quantum system by coupling the two end spins, at site 1 on the left end, $L$, and at site $N$ on the right end, $R$, to two independent free bosonic thermal baths with Hamiltonians

$$
H_{\alpha}=\int_{0}^{+\infty} d \nu v \mathfrak{b}_{\alpha}^{\dagger}(v) \mathfrak{b}_{\alpha}(v), \quad \alpha=L, R,
$$

where $\mathfrak{b}_{\alpha}(v), \mathfrak{b}_{\alpha}^{\dagger}(v)$ are bosonic operators satisfying the canonical commutation relations

$$
\left[\mathfrak{b}_{\alpha}(v), \mathfrak{b}_{\beta}^{\dagger}\left(v^{\prime}\right)\right]=\delta_{\alpha \beta} \delta\left(v-v^{\prime}\right)
$$

The coupling of the baths to the left and right spins is described by the interaction Hamiltonian

$$
H^{\prime}=\lambda \sum_{\alpha=L, R}\left(\sigma_{+}^{(\alpha)} \mathfrak{B}_{\alpha}+\sigma_{-}^{(\alpha)} \mathfrak{B}_{\alpha}^{\dagger}\right)
$$

where $\lambda \ll 1$ is a dimensionless coupling constant,

$$
\sigma_{ \pm}^{(\ell)} \equiv \frac{1}{2}\left(\sigma_{x}^{(\ell)} \pm i \sigma_{y}^{(\ell)}\right)
$$

are spin-ladder operators at site $\ell$ and hence $\sigma_{ \pm}^{(L)}=\sigma_{ \pm}^{(1)}$ and $\sigma_{ \pm}^{(R)}=\sigma_{ \pm}^{(N)}$, while

$$
\mathfrak{B}_{\alpha}=\int_{0}^{\infty} d \nu h_{\alpha}(v) \mathfrak{b}_{\alpha}(v), \quad\left[h_{\alpha}(v)\right]^{*}=h_{\alpha}(\nu),
$$

are bath operators, with $*$ denoting complex conjugation, and $h_{L, R}(v)$ are suitable smearing functions.

Referring to Ref. [50] for more details, in the first of the following two subsections we shortly review the rigorous weak-coupling-limit derivation of the open-chain master equation of GKSL type in the so-called global approach. It asks for the diagonalization of the full-chain Hamiltonian, so in the second subsection we find the $N$-spin energy eigenvalues and eigenvectors. As we show, the resulting dissipative dynamics of the $N$ spins in the presence of the two baths involves collective spin operators.

\section{A. Dissipative chain dynamics in the weak-coupling limit}

If we assume the free boson baths to be in their equilibrium Gibbs states at temperatures $T_{L} \equiv 1 / \beta_{L}$ and $T_{R} \equiv$ $1 / \beta_{R}$, the state of the environment is then given by

$$
\rho_{\mathrm{env}}=\frac{e^{-\beta_{L} H_{L}}}{\operatorname{Tr}\left(e^{-\beta_{L} H_{L}}\right)} \otimes \frac{e^{-\beta_{R} H_{R}}}{\operatorname{Tr}\left(e^{-\beta_{R} H_{R}}\right)} .
$$

It is invariant under the bath dynamics generated by $H_{\text {env }}=$ $\sum_{\alpha=L, R} H_{\alpha}$ and exhibits thermal expectations of the form

$$
\begin{aligned}
& \operatorname{Tr}_{B}\left(\rho_{\mathrm{env}} b_{\alpha}^{\dagger}(v) b_{\alpha^{\prime}}\left(v^{\prime}\right)\right)=\delta_{\alpha \alpha^{\prime}} \delta\left(v-v^{\prime}\right) n_{\alpha}(v) \\
& \operatorname{Tr}_{B}\left(\rho_{\mathrm{env}} b_{\alpha}(v) b_{\alpha^{\prime}}^{\dagger}\left(v^{\prime}\right)\right)=\delta_{\alpha \alpha^{\prime}} \delta\left(v-v^{\prime}\right)\left(1+n_{\alpha}(v)\right),
\end{aligned}
$$

with thermal mean-occupation numbers

$$
n_{\alpha}(v)=\frac{1}{e^{\beta_{\alpha} v}-1}, \quad v \geq 0 .
$$

Finally, choosing the initial state of the compound system chain plus baths of the form $\rho_{\text {tot }}(0)=\rho(0) \otimes \rho_{\text {env }}$, where $\rho(0)$ is an initial state of the $N$ spins of the chain, in the presence of thermal correlation functions decaying on a time scale much faster than the one typical of the spin chain, one applies the weak-coupling-limit techniques and obtains fully physically consistent, namely completely positive, dissipative chain dynamics [22-37]. In practice, the initial state of the compound system spin chain plus baths evolves into

$$
\rho_{\text {tot }}(t)=e^{-i t H_{\text {tot }}} \rho_{\text {tot }}(0) e^{i t H_{\text {tot }}},
$$

where $H_{\text {tot }}=H+H_{\text {env }}+H^{\prime}$ is the total system Hamiltonian. The state of the open chain at time $t, \rho(t)$, is then 
retrieved by tracing over the degrees of freedom of the baths, $\rho(t)=\operatorname{Tr}_{\text {env }}\left(\rho_{\text {tot }}(t)\right)$. Then, one rescales the physical time variable $t$ to $\tau=t \lambda^{2}$ and takes the limit $\lambda \rightarrow 0$ in $\partial_{t} \operatorname{Tr}_{\text {env }}\left(\rho_{\text {tot }}(t)\right)$. In doing so, oscillations in time due to nonzero differences between chain transition frequencies $E_{i}-E_{j}$ are suppressed. Here, $E_{i}$ and $\left|E_{i}\right\rangle$ solve the spin Hamiltonian eigenvalue equation $H\left|E_{i}\right\rangle=E_{i}\left|E_{i}\right\rangle$ and the energy differences are denoted by $\omega$ in the following. This procedure corresponds to the so-called rotating-wave approximation, leading to a master equation of the GKSL form:

$$
\frac{\partial \rho(t)}{\partial t}=-i\left[H+\lambda^{2} H_{\mathrm{LS}}, \rho(t)\right]+\mathbb{D}[\rho(t)]=\mathbb{L}[\rho(t)] .
$$

On the right-hand side of the above time-evolution equation, one distinguishes a Hamiltonian term $\lambda^{2} H_{\mathrm{LS}}$, which provides a Lamb-shift correction to the spin-chain Hamiltonian $H$, and a purely dissipative term $\mathbb{D}[\rho(t)]$. As we see, in the specific physical context here considered, $\mathbb{D}[\rho(t)]$ consists of contributions $\mathbb{D}_{\omega}^{(\alpha)}[\rho(t)]$ resulting from positive transition frequencies $\omega \geq 0$ only:

$$
\mathbb{D}[\rho(t)]=\lambda^{2} \sum_{\alpha=L, R} \sum_{\omega \geq 0} \mathbb{D}_{\omega}^{(\alpha)}[\rho(t)] .
$$

Their explicit form reads

$$
\begin{aligned}
\mathbb{D}_{\omega}^{(\alpha)} & {[\rho(t)] } \\
& =C_{\omega}^{(\alpha)}\left[A_{\alpha}(\omega) \rho(t) A_{\alpha}^{\dagger}(\omega)-\frac{1}{2}\left\{A_{\alpha}^{\dagger}(\omega) A_{\alpha}(\omega), \rho(t)\right\}\right] \\
& +\widetilde{C}_{\omega}^{(\alpha)}\left[A_{\alpha}^{\dagger}(\omega) \rho(t) A_{\alpha}(\omega)-\frac{1}{2}\left\{A_{\alpha}(\omega) A_{\alpha}^{\dagger}(\omega), \rho(t)\right\}\right],
\end{aligned}
$$

the coefficients of which,

$$
\begin{aligned}
& C_{\omega}^{(\alpha)}=2 \pi\left[h_{\alpha}(\omega)\right]^{2}\left(n_{\alpha}(\omega)+1\right), \\
& \widetilde{C}_{\omega}^{(\alpha)}=2 \pi\left[h_{\alpha}(\omega)\right]^{2} n_{\alpha}(\omega),
\end{aligned}
$$

with $n_{\alpha}(\omega)$ as in Eq. (9), come from the real parts of the half-Fourier transforms of the bath correlation functions.

Remark 1: Note that the anticommutators in the above expressions can be interpreted as damping terms, effectively contributing with anti-Hermitian terms to the chain Hamiltonian. Under their action, $\operatorname{Tr}\left(\rho_{t}\right)$ decreases and hence they operate as probability sinks. The remaining terms are instead noise terms that counteract the damping ones acting as probability sources that make the overall probability constant, $\operatorname{Tr}\left(\rho_{t}\right)=1$ for all $t \geq 0$.
Instead, the Lamb-shift correction amounts to the Hamiltonian

$$
H_{\mathrm{LS}}=\sum_{\alpha=L, R} \sum_{\omega}\left[S_{\omega}^{(\alpha)} A_{\alpha}^{\dagger}(\omega) A_{\alpha}(\omega)+\widetilde{S}_{\omega}^{(\alpha)} A_{\alpha}(\omega) A_{\alpha}^{\dagger}(\omega)\right],
$$

where, unlike the dissipative term, the sum now runs over all positive and negative transition frequencies and the coefficients of which read

$$
\begin{aligned}
& S_{\omega}^{(\alpha)}=P \int_{0}^{+\infty} d v\left[h_{\alpha}(v)\right]^{2} \frac{1+n_{\alpha}(v)}{\omega-v}, \\
& \widetilde{S}_{\omega}^{(\alpha)}=P \int_{0}^{+\infty} d v\left[h_{\alpha}(v)\right]^{2} \frac{n_{\alpha}(v)}{v-\omega},
\end{aligned}
$$

where $P$ denotes the principal value.

In all the previous expressions, there appear Lindblad operators of the form

$$
A_{\alpha}^{\dagger}(\omega)=\sum_{E_{i}-E_{j}=\omega}\left|E_{i}\right\rangle\left\langle E_{i}\left|\sigma_{+}^{(\alpha)}\right| E_{j}\right\rangle\left\langle E_{j}\right|,
$$

together with their Hermitian conjugates

$$
A_{\alpha}(\omega)=\sum_{E_{i}-E_{j}=\omega}\left|E_{j}\right\rangle\left\langle E_{j}\left|\sigma_{-}^{(\alpha)}\right| E_{i}\right\rangle\left\langle E_{i}\right| .
$$

In order to obtain explicit expressions for the elements of the master equation (11), one needs to work with eigenvalues and eigenvectors of the full spin Hamiltonian $H$ : this point of view is known as the global approach to open quantum spin chains. This way of proceeding contrasts with the so-called local approach, where the weakcoupling limit is implemented by switching off the spin interactions, thus obtaining strictly local dissipative terms that involve only the left and the right spins. The spin interactions are then reinserted at the end of the weak-coupling procedure.

Remark 2: The fact that the dissipative contribution to the generator, $\mathbb{D}[\rho(t)]$, involves only transition frequencies $\omega \geq 0$ is due the thermal bath energies being positive and to the form of the interaction in Eq. (3). Indeed, in the interaction representation, terms such as $A_{\alpha}^{\dagger}(\omega) \mathfrak{b}_{\alpha}(v)$ contribute with time oscillations $\exp ( \pm i(v-\omega) t)$. On the time scale $\tau=t / \lambda^{2}$ and in the weak-coupling limit when $\lambda \rightarrow 0$, fast oscillations select contributions with $\omega=v \geq$ 0 . Negative transition frequencies, $\omega \leq 0$, would also be selected if in Eq. (3) there were interaction terms of the form $\sigma_{+}^{(\alpha)} \mathfrak{b}_{\alpha}^{\dagger}(v)$, which, together with their Hermitian conjugates, would correspond to the presence of terms of the form $A_{\alpha}^{\dagger}(\omega) \mathfrak{b}_{\alpha}^{\dagger}(v)$, and Hermitian conjugates, contributing with time oscillations $\exp ( \pm i(v+\omega) t)$. 


\section{B. Spin-chain Hamiltonian: eigenvalues and eigenvectors}

In order to address how the presence of the baths modifies the chain dynamics in the weak-coupling limit and within the global approach, we first need to diagonalize the chain Hamiltonian in Eq. (1). By means of the $\ell$ th spin-ladder operators in Eq. (4), one rewrites as follows:

$$
H=\Delta \sum_{\ell=1}^{N} \sigma_{z}^{(\ell)}+2 g \sum_{\ell=1}^{N-1}\left(\sigma_{+}^{(\ell)} \sigma_{-}^{(\ell+1)}+\sigma_{-}^{(\ell)} \sigma_{+}^{(\ell+1)}\right) .
$$

By means of the Jordan-Wigner transformation [51], one introduces fermionic annihilation and creation operators:

$$
a_{j}:=\prod_{k=1}^{j-1}\left(-\sigma_{z}^{(k)}\right) \sigma_{-}^{(j)}, \quad a_{j}^{\dagger}=\prod_{k=1}^{j-1}\left(-\sigma_{z}^{(k)}\right) \sigma_{+}^{(j)},
$$

with the convention that $\prod_{k=1}^{j-1}\left(-\sigma_{z}^{(k)}\right)=1$ for $j=1$, satisfying the anticommutation relations

$$
\left\{a_{j}, a_{k}^{\dagger}\right\}=\delta_{j k} .
$$

Let $|\uparrow\rangle$ and $|\downarrow\rangle$ be the eigenvectors of $\sigma_{z}, \sigma_{z}|\uparrow\rangle=|\uparrow\rangle$ and $\sigma_{z}|\downarrow\rangle=-|\downarrow\rangle$. Since $\sigma_{-}|\downarrow\rangle=0$, the vacuum vector such that $a_{j}|v\rangle=0$, for all $j=1,2, \ldots, N$, amounts to

$$
|v\rangle=|\downarrow\rangle^{\otimes N} .
$$

Using $a_{j}^{\dagger} a_{j}=\sigma_{+}^{(j)} \sigma_{-}^{(j)}=\left(1^{(j)}+\sigma_{z}^{(j)}\right) / 2$, one inverts the transformation given in Eq. (23):

$$
\sigma_{-}^{(j)}=\left(\sigma_{+}^{(j)}\right)^{\dagger}=\prod_{k=1}^{j-1}\left(1-2 a_{k}^{\dagger} a_{k}\right) a_{j}, \quad \sigma_{z}^{(j)}=2 a_{j}^{\dagger} a_{j}-1,
$$

finally turning the spin Hamiltonian into a fermionic one, $H=-N \Delta+2 g \widetilde{H}$, where

$$
\widetilde{H}=\gamma \sum_{j=1}^{N} a_{j}^{\dagger} a_{j}+\sum_{j=1}^{N-1}\left(a_{j}^{\dagger} a_{j+1}+a_{j+1}^{\dagger} a_{j}\right), \quad \gamma:=\frac{\Delta}{g} .
$$

As shown in Appendix A, $H$ can then be diagonalized:

$$
H=-N \Delta+\sum_{\ell=1}^{N}\left(2 \Delta+4 g \cos \left(\frac{\ell \pi}{N+1}\right)\right) b_{\ell}^{\dagger} b_{\ell},
$$

where the operators

$$
b_{\ell}:=\sum_{j=1}^{N} u_{\ell j} a_{j}, \quad b_{\ell}^{\dagger}:=\sum_{j=1}^{N} u_{\ell j} a_{j}^{\dagger}
$$

are also fermionic, $\left\{b_{j}, b_{k}^{\dagger}\right\}=\delta_{j k}$, with the same vacuum as the operators $a_{j}: b_{\ell}|v\rangle=0$ for all $\ell=1,2, \ldots, N$, while the coefficients

$$
u_{\ell k}=\sqrt{\frac{2}{N+1}} \sin \left(\frac{\ell k \pi}{N+1}\right) .
$$

form an orthogonal and symmetric matrix $U=\left[u_{k \ell}\right]$. In the following, we denote by $\mathbf{n}$ the $N$-tuple $n_{1}, n_{2}, \ldots, n_{N}$, where $n_{j}=0,1$ is the occupation number of the $j$ th mode relative to the operators $b_{j}$ and $b_{j}^{\dagger}$. The eigenvectors of the Hamiltonian given in Eq. (1) have thus the form

$$
|\mathbf{n}\rangle=\left(b_{1}^{\dagger}\right)^{n_{1}}\left(b_{2}^{\dagger}\right)^{n_{2}} \cdots\left(b_{N}^{\dagger}\right)^{n_{N}}|v\rangle .
$$

Indeed, according to Eq. (31),

$$
\begin{aligned}
b_{\ell}|\mathbf{n}\rangle & =\delta_{n_{\ell}, 1}(-1)^{\sum_{j=1}^{\ell-1} n_{j}} \sqrt{n_{\ell}}\left|\mathbf{n}_{\ell}^{-}\right\rangle, \\
b_{\ell}^{\dagger}|\mathbf{n}\rangle & =\delta_{n_{\ell}, 0}(-1)^{\sum_{j=1}^{\ell-1} n_{j}} \sqrt{1-n_{\ell}}\left|\mathbf{n}_{\ell}^{+}\right\rangle \\
b_{\ell}^{\dagger} b_{\ell}|\mathbf{n}\rangle & =n_{\ell}|\mathbf{n}\rangle
\end{aligned}
$$

where, $\mathbf{n}_{\ell}^{ \pm}$denote the $N$-tuples $n_{1}, \ldots, n_{\ell} \pm 1, \ldots, n_{N}$. Then, one verifies that $H|\mathbf{n}\rangle=E_{\mathbf{n}}|\mathbf{n}\rangle$, where

$$
E_{\mathbf{n}}=\Delta\left(2 \sum_{\ell=1}^{N} n_{\ell}-N\right)+4 g \sum_{\ell=1}^{N} n_{\ell} \cos \left(\frac{\ell \pi}{N+1}\right) .
$$

Remark 3: Since the matrix $U=\left[u_{k \ell}\right]$ with entries $u_{k \ell}$ as in Eq. (30) is real and symmetric, from Eq. (29) one obtains

$$
a_{j}=\sum_{\ell=1}^{N} u_{j \ell} b_{\ell}, \quad a_{j}^{\dagger}=\sum_{\ell=1}^{N} u_{j \ell} b_{\ell}^{\dagger} .
$$

Then, by using Eq. (26), one expresses the fermionic operators of type $b$ in terms of spin operators:

$$
\begin{aligned}
& b_{\ell}=\sum_{j=1}^{N} u_{\ell j} \prod_{k=1}^{j-1}\left(-\sigma_{z}^{(k)}\right) \sigma_{-}^{(j)}, \\
& b_{\ell}^{\dagger}=\sum_{j=1}^{N} u_{\ell j} \prod_{k=1}^{j-1}\left(-\sigma_{z}^{(k)}\right) \sigma_{+}^{(j)} .
\end{aligned}
$$

A comparison with known results is provided in Appendix B. 


\section{COUPLING TO EXTERNAL BATHS}

With the notation of the previous section, the Lindblad operators in Eq. (20) now read

$$
A_{\alpha}^{\dagger}(\omega)=\sum_{E_{\mathbf{m}}-E_{\mathbf{n}}=\omega}|\mathbf{m}\rangle\left\langle\mathbf{m}\left|\sigma_{+}^{(\alpha)}\right| \mathbf{n}\right\rangle\langle\mathbf{n}| .
$$

Their explicit form can be derived by expressing the spin operators $\sigma_{+}^{(\alpha)}$ first in terms of the fermionic operators $a_{j}, a_{j}^{\dagger}$

$$
\sigma_{+}^{(L)}=a_{1}^{\dagger}, \quad \sigma_{+}^{(R)}=\prod_{j=1}^{N-1}\left(1-2 a_{j}^{\dagger} a_{j}\right) a_{N}^{\dagger},
$$

and then in terms of the operators $b_{\ell}, b_{\ell}^{\dagger}$. Using Eq. (36), one immediately derives

$$
\sigma_{+}^{(L)}=\sum_{\ell=1}^{N} u_{1 \ell} b_{\ell}^{\dagger}
$$

while the presence of $\prod_{j=1}^{N-1}\left(1-2 a_{j}^{\dagger} a_{j}\right)$ in the expression for $\sigma_{+}^{(R)}$ leads, using $1-2 a_{j}^{\dagger} a_{j}=\exp \left(i \pi a_{j}^{\dagger} a_{j}\right)$ and after straightforward algebraic manipulations, to

$$
\sigma_{+}^{(R)}=-\left(e^{i \pi \sum_{\ell=1}^{N} b_{\ell}^{\dagger} b_{\ell}}\right) \sum_{\ell=1}^{N} u_{N \ell} b_{\ell}^{\dagger} .
$$

By means of Eqs. (41) and (33), one then computes the transition amplitudes

$$
\left\langle\mathbf{m}\left|\sigma_{+}^{(L)}\right| \mathbf{n}\right\rangle=\sum_{\ell=1}^{N}(-1)^{\sum_{j=1}^{\ell-1} n_{j}} \sqrt{1-n_{\ell}} u_{1 \ell} \delta_{\mathbf{m}_{\ell}^{+}} .
$$

Let $\mathbf{n}_{0_{\ell}}$ and $\mathbf{n}_{1_{\ell}}$ denote the $N$-tuples with fixed digits $n_{\ell}=$ 0,1 , respectively, at site $\ell$. Then, the only contributing transition amplitudes are

$$
\left\langle\mathbf{n}_{1_{\ell}}\left|\sigma_{+}^{(L)}\right| \mathbf{n}_{0_{\ell}}\right\rangle=(-1)^{\sum_{j=1}^{\ell-1} n_{j}} \sqrt{1-n_{\ell}} u_{1 \ell},
$$

with $\ell=1,2, \ldots, N$. Also, from Eqs. (35) and (20), the transition frequencies associated with such amplitudes are

$$
\omega_{\ell}=E_{\mathbf{n}_{1_{\ell}}}-E_{\mathbf{n}_{0_{\ell}}}=2 \Delta+4 g \cos \left(\frac{\ell \pi}{N+1}\right),
$$

while the corresponding Lindblad operators in Eq. (39) read

$$
A_{L}^{\dagger}\left(\omega_{\ell}\right)=u_{1 \ell} \sum_{\widehat{\mathbf{n}}_{\ell}}(-1)^{\sum_{j=1}^{\ell-1} n_{j}}\left|\mathbf{n}_{1_{\ell}}\right\rangle\left\langle\mathbf{n}_{0_{\ell}}\right|
$$

where the symbol $\sum_{\widehat{\mathbf{n}}_{\ell}}$ means that the summation is performed over all binary $2^{N-1}$-tuples of indices $n_{j}=0,1$ with $j \neq \ell$. It thus follows that

$$
\begin{aligned}
& A_{L}^{\dagger}\left(\omega_{\ell}\right) A_{L}\left(\omega_{\ell}\right)=u_{1 \ell}^{2} \sum_{\widehat{\mathbf{n}}_{\ell}}\left|\mathbf{n}_{1_{\ell}}\right\rangle\left\langle\mathbf{n}_{1_{\ell}}\right|, \\
& A_{L}\left(\omega_{\ell}\right) A_{L}^{\dagger}\left(\omega_{\ell}\right)=u_{1 \ell}^{2} \sum_{\widehat{\mathbf{n}}_{\ell}}\left|\mathbf{n}_{0_{\ell}}\right\rangle\left\langle\mathbf{n}_{0_{\ell}}\right| .
\end{aligned}
$$

In a similar way, from Eq. (42), one obtains that the only contributing transition amplitudes associated with the right bath are

$$
\left\langle\mathbf{n}_{1_{\ell}}\left|\sigma_{+}^{(R)}\right| \mathbf{n}_{0_{\ell}}\right\rangle=(-1)^{\sum_{j=1}^{\ell+1} n_{j}} \sqrt{1-n_{\ell}} u_{N \ell},
$$

with Lindblad operators

$$
A_{R}^{\dagger}\left(\omega_{\ell}\right)=u_{N \ell} \sum_{\widehat{\mathbf{n}}_{\ell}}(-1)^{\sum_{j=\ell+1}^{N} n_{j}}\left|\mathbf{n}_{1_{\ell}}\right\rangle\left\langle\mathbf{n}_{0_{\ell}}\right|
$$

from which

$$
\begin{aligned}
& A_{R}^{\dagger}\left(\omega_{\ell}\right) A_{R}\left(\omega_{\ell}\right)=u_{N \ell}^{2} \sum_{\widehat{\mathbf{n}}_{\ell}}\left|\mathbf{n}_{1_{\ell}}\right\rangle\left\langle\mathbf{n}_{1_{\ell}}\right|, \\
& A_{R}\left(\omega_{\ell}\right) A_{R}^{\dagger}\left(\omega_{\ell}\right)=u_{N \ell}^{2} \sum_{\widehat{\mathbf{n}}_{\ell}}\left|\mathbf{n}_{0_{\ell}}\right\rangle\left\langle\mathbf{n}_{0_{\ell}}\right| .
\end{aligned}
$$

Note that, in the spin representation, all Lindblad operators $A_{\alpha}^{\dagger}\left(\omega_{\ell}\right)$ involve, through the relations given in Eqs. (26) and (36), products of all on-site spin operators. This structure is typical of the global approach to open spin chains and differs strikingly from the local one, which yields Lindblad operators involving only spin operators pertaining to the first and last spin of the chain. It is worth mentioning that if each spin of the chain were to interact with an independent thermal bath by means of a Hamiltonian similar to Eq. (3), then for deriving the Lindblad operators, in addition to the matrix elements of the form $\sigma_{+}^{(L / R)}$ in Eqs. (43) and (49), it would be essential to also compute the matrix elements of the ladder operators $\sigma_{ \pm}^{(\ell)}$ at all sites $\ell$ [52].

The operators $A_{\alpha}\left(\omega_{\ell}\right)$ and $A_{\alpha}^{\dagger}\left(\omega_{\ell}\right), \alpha=L, R$, have to be inserted into the expressions Eqs. (13) and (14) when $\omega=\omega_{\ell} \geq 0$, together with $C_{\omega_{\ell}}^{(\alpha)}$ and $\widetilde{C}_{\omega_{\ell}}^{(\alpha)}$ as in Eqs. (15) and (16). Instead, the Lamb-shift Hamiltonian given in Eq. (17), contributing to Eq. (11), requires the operators $A_{\alpha}^{\dagger}\left(\omega_{\ell}\right) A_{\alpha}\left(\omega_{\ell}\right)$ and $A_{\alpha}\left(\omega_{\ell}\right) A_{\alpha}^{\dagger}\left(\omega_{\ell}\right), \alpha=L, R$, with both positive and negative $\omega_{\ell}$. The Hamiltonian $H+\lambda^{2} H_{\mathrm{LS}}$ in Eq. (11) is thus diagonal in the energy eigenbasis $\{|\mathbf{n}\rangle\}$.

The $N=2$ and $N=3$ cases are explicitly worked out in Appendix C.

Remark 4: Some observations are in order at this point. The first is that, as a consequence of the fact that the transition frequencies contributing to the dissipative generator 
in Eq. (12) are positive, not all those corresponding to the nonvanishing Lindblad operators $A_{\alpha}^{\dagger}\left(\omega_{\ell}\right)$ in Eqs. (46) and (50) need be such. This means that the Lindblad operators $A_{\alpha}^{\dagger}\left(\omega_{\ell}\right)$ with $\omega_{\ell}<0$ can only contribute to the Lamb-shift Hamiltonian and not to the dissipative part of the generator. The sign of $\omega_{\ell}$ depends on the strength of the interspin coupling constant g; indeed,

$$
\cos \left(\frac{\pi \ell}{N+1}\right)<0 \text { for } N \geq \ell>\frac{N+1}{2} .
$$

Correspondingly, $\omega_{\ell}<0$ for $g>\Delta / 2|\cos [\ell \pi /(N+1)]|$.

The second observation is that should any of the transition frequencies $\omega_{\ell}$ in the list $E q$. (45) be negative, the opposite one, $-\omega_{\ell}=E_{\mathbf{n}_{0_{\ell}}}-E_{\mathbf{n}_{\mathbf{n}_{\ell}}}$, not being in the list, would not give rise to a dissipative contribution of the form $\mathbb{D}_{-\omega_{\ell}}[\rho(t)]$, as $A_{\alpha}^{\dagger}\left(-\omega_{\ell}\right) \equiv 0$. In the following, we assume that

$$
g \leq \frac{\Delta}{2 \cos [\pi /(N+1)]},
$$

so that $\omega_{\ell} \geq 0, \ell=1,2, \ldots, N$, and leave the study of the presence of negative transition frequencies for future investigations.

\section{STATIONARY STATE}

The master equation given in Eq. (11) possesses a unique stationary state that is left invariant by the generated reduced dynamics, namely such that $\mathbb{L}\left[\rho_{\infty}\right]=0$. This follows from the fact that, as shown in Appendix D 1, the only operator commuting with all Lindblad operators $A_{L, R}\left(\omega_{\ell}\right)$ and $A_{L, R}^{\dagger}\left(\omega_{\ell}\right)$ and with the Hamiltonian must be a multiple of the identity [53-58].

As explained before Remark $4, H+\lambda^{2} H_{\mathrm{LS}}$ is diagonal in the energy eigenbasis $|\mathbf{k}\rangle$. Therefore, the Hamiltonian contribution to the generator given in Eq. (11) yields $\left[H+\lambda^{2} H_{\mathrm{LS}}, P_{\mathbf{k}}\right]=0$, for all energy eigenprojections $P_{\mathbf{k}}:=|\mathbf{k}\rangle\langle\mathbf{k}|$. In Appendix D2, it is shown that the action of the dissipative part of the generator on the eigenprojetions yields a unique stationary state of the form

$$
\begin{gathered}
\rho_{\infty}=\sum_{\mathbf{n}} \Lambda_{\mathbf{n}} P_{\mathbf{n}}, \quad \Lambda_{\mathbf{n}}=\prod_{\ell=1}^{N} \lambda_{n_{\ell}}^{(\ell)}, \\
\lambda_{n_{\ell}}^{(\ell)}=\frac{R_{n_{\ell}}^{(\ell)}}{R_{\ell}}, \\
R_{n_{\ell}}^{(\ell)}:=\left[h_{L}\left(\omega_{\ell}\right)\right]^{2}\left(1-n_{\ell}+n_{L}\left(\omega_{\ell}\right)\right) \\
+\left[h_{R}\left(\omega_{\ell}\right)\right]^{2}\left(1-n_{\ell}+n_{R}\left(\omega_{\ell}\right)\right),
\end{gathered}
$$

$R_{\ell}:=\left[h_{L}\left(\omega_{\ell}\right)\right]^{2}\left(1+2 n_{L}\left(\omega_{\ell}\right)\right)+\left[h_{R}\left(\omega_{\ell}\right)\right]^{2}\left(1+2 n_{R}\left(\omega_{\ell}\right)\right)$.

With the simplifying assumption $h_{L, R}\left(\omega_{\ell}\right)=h$, for each $\ell=1,2, \ldots, N$, one retrieves

$$
\lambda_{n_{\ell}}^{(\ell)}=\frac{1}{2}\left[1+\frac{(-1)^{n_{\ell}}}{1+n_{L}\left(\omega_{\ell}\right)+n_{R}\left(\omega_{\ell}\right)}\right] .
$$

If we further restrict to identical baths, by imposing equal temperatures and thus $\beta_{L}=\beta_{R}=\beta$, one computes

$$
\lambda_{n_{\ell}}^{(\ell)}=\frac{e^{\beta\left(1-n_{\ell}\right) \omega_{\ell}}}{e^{\beta \omega_{\ell}}+1},
$$

so that

$$
\rho_{\infty}=\sum_{\mathbf{n}} \prod_{\ell=1}^{N} \frac{e^{\beta\left(1-n_{\ell}\right) \omega_{\ell}}}{e^{\beta \omega_{\ell}}+1}|\mathbf{n}\rangle\langle\mathbf{n}| .
$$

On the other hand, using Eq. (35),

$$
\frac{e^{-\beta E_{\mathbf{n}}}}{\sum_{\mathbf{n}} e^{-\beta E_{\mathbf{n}}}}=\prod_{\ell=1}^{N} \frac{e^{2 \beta\left(1-n_{\ell}\right)}\{\Delta+2 g \cos [\pi \ell /(N+1)]\}}{e^{2 \beta\left(1-n_{\ell}\right)}\{\Delta+2 g \cos [\pi \ell /(N+1)]}+1 .
$$

Then, Eq. (45) implies that the open-chain stationary state $\rho_{\infty}$ is the Gibbs state at inverse temperature $\beta$ with Hamiltonian $H$ as given in Eq. (1):

$$
\rho_{\infty}=\frac{e^{-\beta H}}{\operatorname{Tr}\left(e^{-\beta H}\right)}
$$

\section{TRANSPORT PROPERTIES}

Having determined the explicit analytic form of the stationary state, we can now study its transport properties by analyzing the spin and heat flows along the chain, driven by the two external baths.

\section{A. Stationary spin flow: Sinks and sources}

The spin flow at site $k=1,2, \ldots, N$ along the spin chain corresponds to the rate of change in time of the average of $\sigma_{z}^{(k)}$ given by the quantity

$$
\begin{aligned}
\frac{d}{d t} \operatorname{Tr}\left[\sigma_{z}^{(k)} \rho(t)\right] & =\operatorname{Tr}\left[\sigma_{z}^{(k)} \mathbb{L}[\rho(t])\right] \\
& =\operatorname{Tr}\left[\widetilde{\mathbb{L}}\left[\sigma_{z}^{(k)}\right] \rho(t)\right]
\end{aligned}
$$


In the first equality, $\mathbb{L}$ is the generator on the right-hand side of Eq. (11), while the second equality follows from the cyclicity of the trace, $\operatorname{Tr}(X Y)=\operatorname{Tr}(Y X)$ and defines the so-called dual generator $\widetilde{\mathbb{L}}$ :

$$
\tilde{\mathbb{L}}\left[\sigma_{z}^{(k)}\right]=i\left[H+\lambda^{2} H_{\mathrm{LS}}, \sigma_{z}^{(k)}\right]+\widetilde{\mathbb{D}}\left[\sigma_{z}^{(k)}\right],
$$

with $\widetilde{\mathbb{D}}\left[\sigma_{z}^{(k)}\right]=\lambda^{2} \sum_{\alpha=L, R} \sum_{\omega_{\ell} \geq 0}^{N} \widetilde{\mathbb{D}}_{\omega_{\ell}}^{(\alpha)}\left[\sigma_{z}^{(k)}\right]$, where

$$
\begin{aligned}
\tilde{\mathbb{D}}_{\omega_{\ell}}^{(\alpha)} & {\left[\sigma_{z}^{(k)}\right] } \\
& =C_{\omega_{\ell}}^{(\alpha)}\left[A_{\alpha}^{\dagger}\left(\omega_{\ell}\right) \sigma_{z}^{(k)} A_{\alpha}\left(\omega_{\ell}\right)-\frac{1}{2}\left\{A_{\alpha}^{\dagger}\left(\omega_{\ell}\right) A_{\alpha}\left(\omega_{\ell}\right), \sigma_{z}^{(k)}\right\}\right] \\
& +\widetilde{C}_{\omega_{\ell}}^{(\alpha)}\left[A_{\alpha}\left(\omega_{\ell}\right) \sigma_{z}^{(k)} A_{\alpha}^{\dagger}\left(\omega_{\ell}\right)-\frac{1}{2}\left\{A_{\alpha}\left(\omega_{\ell}\right) A_{\alpha}^{\dagger}\left(\omega_{\ell}\right), \sigma_{z}^{(k)}\right\}\right] .
\end{aligned}
$$

The Hamiltonian contribution to the rate of change in time of the average of $\sigma_{z}$ can be expressed in terms of the dimensionless spin currents:

$$
J^{(k, k+1)}=4 i\left(\sigma_{-}^{(k)} \sigma_{+}^{(k+1)}-\sigma_{+}^{(k)} \sigma_{-}^{(k+1)}\right),
$$

as

$$
i\left[H+\lambda^{2} H_{\mathrm{LS}}, \sigma_{z}^{(k)}\right]=(g+\kappa)\left(J^{(k-1, k)}-J^{(k, k+1)}\right),
$$

where the Lamb-shift contribution is characterized by a constant:

$$
\kappa=\frac{i \lambda^{2}}{8 \sqrt{2}} \sum_{\alpha=L, R} \sum_{\ell=1}^{N}\left(S_{\omega_{\ell}}^{(\alpha)}-\widetilde{S}_{\omega_{\ell}}^{(\alpha)}\right) .
$$

The operator differences in Eq. (68) thus contribute to the continuity equation Eq. (63) as current divergence terms with the right dimension of energy. Since we are interested in the stationary transport properties, we set $\rho(t)=\rho_{\infty}$ on the right-hand side of Eq. (63) and find $\left\langle J^{(k, k+1)}\right\rangle_{\infty}:=\operatorname{Tr}\left(\rho_{\infty} J^{(k, k+1)}\right)=0$. Indeed, passing from spin to fermionic operators by means of Eqs. (26) and (36), one finds that

$$
\begin{aligned}
J^{(k, k+1)} & =-4 i\left(a_{k} a_{k+1}^{\dagger}+a_{k}^{\dagger} a_{k+1}\right) \\
& =-4 i \sum_{j \ell=1}^{N} u_{k j} u_{k+1 \ell}\left(b_{j} b_{\ell}^{\dagger}+b_{j}^{\dagger} b_{\ell}\right) .
\end{aligned}
$$

Hence, all their averages with respect to the energy eigenstates vanish:

$$
\left\langle\mathbf{n}\left|J^{(k, k+1)}\right| \mathbf{n}\right\rangle=-4 i \sum_{j=1}^{N} u_{k j} u_{k+1 j}=\left\langle u_{k} \mid u_{k+1}\right\rangle=0 .
$$

Indeed, Eqs. (32) and (33) yield $\left\langle\mathbf{n}\left|b_{j} b_{\ell}^{\dagger}\right| \mathbf{n}\right\rangle=\delta_{j \ell}\left(1-n_{j}\right)$ and $\left\langle\mathbf{n}\left|b_{j}^{\dagger} b_{\ell}\right| \mathbf{n}\right\rangle=\delta_{j \ell} n_{j}$, while the columns $\left|u_{k}\right\rangle$ of the orthogonal and symmetric matrix $U$ (see Remark 3 ) are orthogonal. Thus the stationary left, $\left\langle J^{(k-1, k)}\right\rangle_{\infty}$, and right, $\left\langle J^{(k, k+1)}\right\rangle_{\infty}$, spin currents through site $k$ both vanish.

Clearly, $\rho_{\infty}$ being time independent, the right-hand side of Eq. (63) then yields $\operatorname{Tr}\left(\rho_{\infty} \mathbb{D}\left[\sigma^{(k)}\right]\right)=0$. However, the left and right purely dissipative contributions,

$$
\mathfrak{Q}_{\alpha}^{(k)}:=\lambda^{2} \sum_{\ell=1}^{N} \operatorname{Tr}\left(\rho_{\infty} \widetilde{\mathbb{D}}_{\omega_{\ell}}^{(\alpha)}\left[\sigma_{z}^{(k)}\right]\right),
$$

do not vanish separately; indeed, as shown in Appendix E,

$$
\begin{aligned}
\mathfrak{Q}_{L}^{(k)}= & 2 \pi \lambda^{2} \sum_{\ell=1}^{N} u_{k \ell}^{2} u_{1 \ell}^{2}\left[h_{L}\left(\omega_{\ell}\right)\right]^{2}\left[h_{R}\left(\omega_{\ell}\right)\right]^{2} \\
& \times \frac{n_{L}\left(\omega_{\ell}\right)-n_{R}\left(\omega_{\ell}\right)}{R_{\ell}} \\
\mathfrak{Q}_{R}^{(k)}= & 2 \pi \lambda^{2} \sum_{\ell=1}^{N} u_{k \ell}^{2} u_{N \ell}^{2}\left[h_{L}\left(\omega_{\ell}\right)\right]^{2}\left[h_{R}\left(\omega_{\ell}\right)\right]^{2} \\
& \times \frac{n_{R}\left(\omega_{\ell}\right)-n_{L}\left(\omega_{\ell}\right)}{R_{\ell}}
\end{aligned}
$$

with $R_{\ell}$ as in Eq. (57). Furthermore, since from Eq. (30) one finds that $u_{1 \ell}=(-1)^{\ell} u_{N \ell}$ for all $\ell=1,2, \ldots, N$, it follows that $\mathfrak{Q}_{L}^{(k)}=-\mathfrak{Q}_{R}^{(k)}$, as it should be in physical terms.

Also, assuming $h_{L}(\omega)=h_{R}(\omega)=h$, one obtains

$$
\begin{aligned}
& \mathfrak{Q}_{L}^{(k)}=\pi \lambda^{2} \sum_{\ell=1}^{N} u_{k \ell}^{2} u_{1 \ell}^{2} \frac{n_{L}\left(\omega_{\ell}\right)-n_{R}\left(\omega_{\ell}\right)}{1+n_{L}\left(\omega_{\ell}\right)+n_{R}\left(\omega_{\ell}\right)}, \\
& \mathfrak{Q}_{R}^{(k)}=\pi \lambda^{2} \sum_{\ell=1}^{N} u_{k \ell}^{2} u_{N \ell}^{2} \frac{n_{R}\left(\omega_{\ell}\right)-n_{L}\left(\omega_{\ell}\right)}{1+n_{L}\left(\omega_{\ell}\right)+n_{R}\left(\omega_{\ell}\right)} .
\end{aligned}
$$

One thus sees that, while in the stationary case the continuity expression given in Eq. (63) does not contain any current divergence at site $k$, it does, however, contain terms of a different origin that are due to the presence of the two baths. These terms vanish only if the temperatures are the same, so that $n_{L}\left(\omega_{\ell}\right)=n_{R}\left(\omega_{\ell}\right)$, and are thus interpretable as spin flow source or sink contributions, depending on whether they are positive or negative. Note that due to the scaling as $1 / N^{2}$ of the products $u_{k \ell}^{2} u_{N \ell}^{2}$ (see Eq. (30)) and the presence of $N$ of them in Eqs. (75) and (76), the source and sink terms scale as $1 / N$ with an increasing number of spins. In Fig. 1, we consider a chain with $N=8$ spins, set $T_{L}=0$ so that $n_{L}\left(\omega_{\ell}\right)=0$, and show the dependence of 


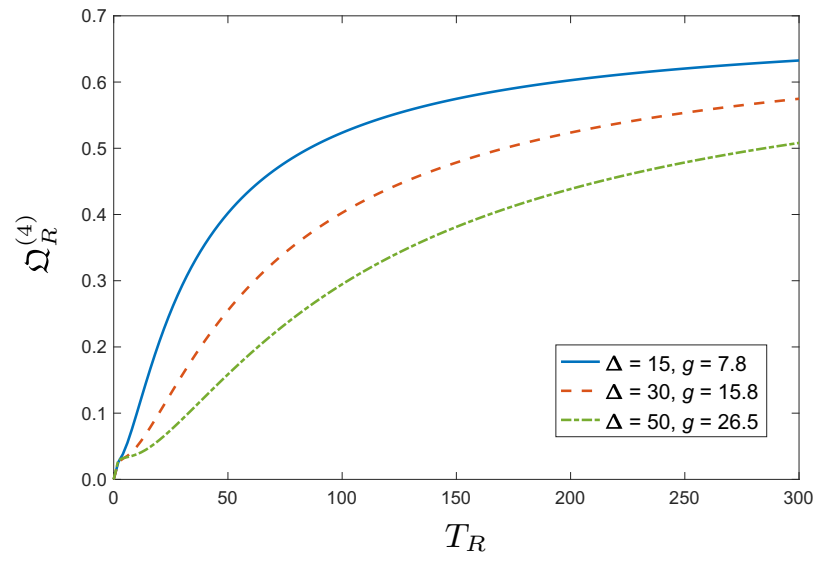

FIG. 1. The behavior of the source term $\mathfrak{Q}_{R}^{(4)}$ as a function of $T_{R}$ in a $N=8$ spin chain with left-bath temperature $T_{L}=10, \lambda=$ $1, \Delta=15,30,50$, and $g$ close to the saturation values in Eq. (53).

the source term

$$
\mathfrak{Q}_{R}^{(4)}=\pi \lambda^{2} \sum_{\ell=1}^{8} u_{4 \ell}^{2} u_{8 \ell}^{2} e^{-\beta_{R} \omega_{\ell}},
$$

in the middle of the chain as a function of the right temperature $T_{R}$ and various values of the transverse magnetic field $\Delta$ and the interspin coupling strength $g$. The values of $g$ associated with $\Delta$ are chosen close to the bound given in Eq. (53), for reasons that will become clear later, when we discuss the bipartite stationary entanglement.

The presence of sink and source terms in the continuity equation of quantum systems can be phenomenologically achieved by means of inserting anti-Hermitian terms in the system Hamiltonian. As already commented in Remark 1, this is exactly what the anticommutators do in the dissipative part of the generator. In some particular cases - as, for instance, in the charge-continuity equation for open systems with local system-bath interactions [59] - the damping and noise terms together contribute to form the divergence of a dissipative current. This is not the case for the open quantum spin chain investigated in this paper, where only the end spins interact with the thermal baths. Here, dissipative currents do not emerge in the continuity equation; rather, sink and source contributions appear that are strictly related to the global structure of the Lindblad operators in Eqs. (46) and (50), which involve all spins of the chain. Indeed, the chain degrees of freedom that are relevant to expressing how the spins react to the presence of the two baths are the fermionic ones, which diagonalize the chain Hamiltonian and are delocalized along it. The roots of the sink and source terms lie in the global features of the damping and noise effects embodied by the anticommutator and the noise term in Eqs. (13) and (14). That it is indeed so is highlighted: (1) by the fact that, should there be no fermionic dressing of the Lindblad operators as in the local approach to open spin chains (see Remark 4), the sink and source terms would disappear, as is the case for the two spins in Ref. [60]; and (2) by the fact that in the global approach, sinks and sources are present even in the limit where the interspin coupling $g \rightarrow 0$. Indeed, $g$ appears in the thermal factors $n_{L, R}\left(\omega_{\ell}\right)$ through the transition frequencies $\omega_{\ell}$ (see Eq. (9)). These terms remain different and nonzero whenever $\beta_{L} \neq \beta_{R}$, even for $g=0$. This evidence shows a dramatic departure of the global approach from the local one, in the sense that the latter cannot be obtained as the $g \rightarrow 0$ limit of the former. Furthermore, this feature together with the asymptotic presence of sinks and sources provide a means of discriminating, through the experimental investigation of the spin polarization at a given site, which one of the two approaches is more appropriate to describe the physics of open quantum spin chains. Since, asymptotically, sinks and sources come together and cancel each other, their presence should be experimentally accessed via an investigation of the polarization transient behavior - the theoretical description of which is, however, beyond the scope of the present work.

\section{B. Stationary heat flow}

Besides the spin flow, the presence of the two baths at the far ends of the chain also establishes heat flows in and out of the chain. According to standard quantum thermodynamics arguments $[39,40]$, the heat flow through an open quantum system due to its weak coupling to a thermal bath is measured by

$$
\mathfrak{H}(t):=\operatorname{Tr}\left(\frac{d \rho(t)}{d t} H\right)=\operatorname{Tr}(\mathbb{L}[\rho(t)] H),
$$

where $\rho \mapsto \rho(t)$ is the dissipative evolution due to the bath, generated by $\mathbb{L}$, while $H$ is the open-system timeindependent Hamiltonian. Because of the structure of the GKSL equation as in Eq. (11), only the dissipative term of the generator contributes to the heat flow; therefore, in the spin-chain stationary state, the heat flow due to the left, $L$, or right, $R$, bath is given by

$$
\mathfrak{H}_{\alpha}^{\text {st }}=\sum_{\ell=1}^{N} \operatorname{Tr}\left(\mathbb{D}_{\omega_{\ell}}^{(\alpha)}\left[\rho_{\infty}\right] H\right), \quad \alpha=L, R
$$

Certainly, $\mathbb{D}\left[\rho_{\infty}\right]=0$ implies $\mathfrak{H}_{L}^{\text {st }}+\mathfrak{H}_{R}^{\text {st }}=0$; however, as for the spin flow, the single bath contributions to the heat flow need not vanish separately and their sign, if positive, corresponds to heat flowing into the chain from the bath or to heat flowing out of the chain and into the bath. 
Using Eqs. (45), (D2), (54)-(57), (15) and (16), one computes

$$
\begin{aligned}
\mathfrak{H}_{L}^{\mathrm{st}}= & \sum_{\ell=1}^{N} \operatorname{Tr}\left(\mathbb{D}_{\omega_{\ell}}^{(L)}\left[\rho_{\infty}\right] H\right)=\lambda^{2} \sum_{\ell=1}^{N} \sum_{\mathbf{n}, \mathbf{k}} E_{\mathbf{n}} \Lambda_{\mathbf{k}} u_{1 \ell}^{2} \\
& \times\left(C_{\omega_{\ell}}^{(L)} \delta_{1 k_{\ell}}-\widetilde{C}_{\omega_{\ell}}^{(L)} \delta_{0 k_{\ell}}\right)\left\langle\mathbf{n}\left|\left(P_{\mathbf{k}_{0_{\ell}}}-P_{\mathbf{k}_{1_{\ell}}}\right)\right| \mathbf{n}\right\rangle \\
= & \lambda^{2} \sum_{\ell=1}^{N} \omega_{\ell} u_{1 \ell}^{2}\left(C_{\omega_{\ell}}^{(L)} \lambda_{1}^{(\ell)}-\widetilde{C}_{\omega_{\ell}}^{(L)} \lambda_{0}^{(\ell)}\right) \\
= & \lambda^{2} \sum_{\ell=1}^{N} \omega_{\ell} u_{1 \ell}^{2}\left[h_{L}\left(\omega_{\ell}\right)\right]^{2}\left[h_{R}\left(\omega_{\ell}\right)\right]^{2} \frac{n_{L}\left(\omega_{\ell}\right)-n_{R}\left(\omega_{\ell}\right)}{R_{\ell}} .
\end{aligned}
$$

Note that the heat flow is positive; namely, it flows from the left bath into the chain if $n_{L}(\omega)>n_{R}(\omega)$, that is (see Eq. (9)), if the left bath is at a higher temperature than the right one. Furthermore, the simplifying assumption $h_{L}(\omega)=h_{R}(\omega)=h$ yields

$$
\mathfrak{H}_{R}^{\mathrm{st}}=\pi \lambda^{2} \sum_{\ell=1}^{N} \omega_{\ell} u_{1 \ell}^{2} \frac{n_{R}\left(\omega_{\ell}\right)-n_{L}\left(\omega_{\ell}\right)}{1+n_{L}\left(\omega_{\ell}\right)+n_{R}\left(\omega_{\ell}\right)} .
$$

Furthermore, the transition frequencies $\omega_{\ell}$ in Eq. (45) are of order 1 with respect to increasing $N$ and hence each of the $N$ contributions $\omega_{\ell} u_{1 \ell}^{2}$ to the heat flow scales as $1 / N$ due to Eq. (30). Thus, unlike the sink and source terms in Eqs. (75) and (76), which scale as $1 / N$, the heat flow does not vanish with increasing $N$. Setting $N=8$ and $T_{L}=0$ as for the source terms in Eq. (77), and choosing the same set of parameters $\Delta$ and $g$ as in Fig. 1, the behavior of the heat flow $\mathfrak{H}_{R}^{\text {st }}$ as a function of $T_{R}$ is reported in Fig. 2.

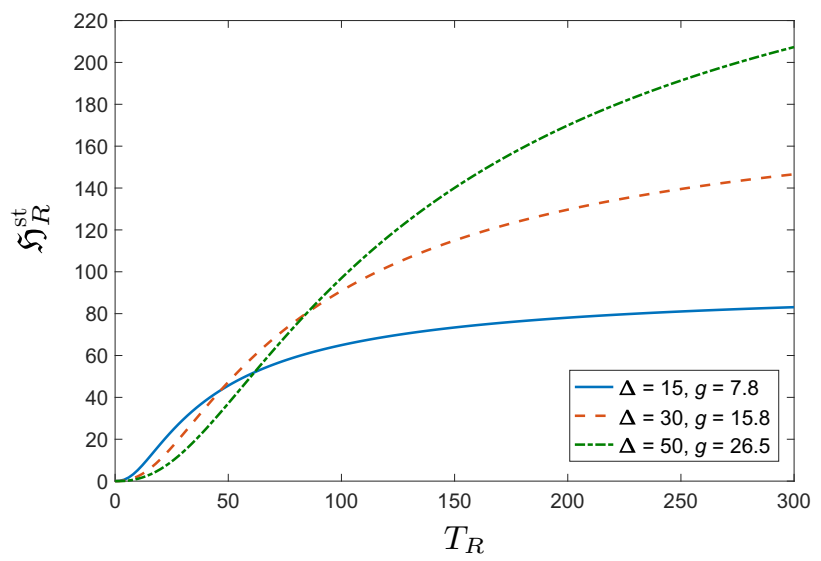

FIG. 2. The behavior of the heat flow $\mathfrak{H}_{R}^{\text {st }}$ as a function of the right-bath temperature $T_{R}$ in a $N=8$ spin chain with left-bath temperature $T_{L}=0, \lambda=1, \Delta=15,30,50$, and $g$ close to the corresponding saturation values.

\section{ENTANGLEMENT PROPERTIES}

Besides transport phenomena, open spin chains represent attractive models of many-body systems due to their entanglement properties. Indeed, although the external transverse magnetic field tends to align all spins in a separable state, the interspin interaction, on the other hand, is able to generate quantum correlations among all spins. The presence of the external baths at the chain end points constitutes interesting additional driving factors influencing the behavior of the spin entanglement.

In what follows, we focus upon the entanglement between any two spins, $r$ and $s>r$, in the stationary state via the concurrence of the reduced bipartite density matrix $\rho_{r, s}$ obtained from Eq. (54) by tracing over the spins at sites different from $r$ and $s$. In order to achieve this goal, one needs to reexpress the stationary state in Eq. (54) in terms of spin operators, rather than fermionic ones.

\section{A. Stationary state: Spin representation}

In order to pass to a spin representation of the stationary state, some preliminary steps are needed. The first is that, instead of the standard lexicographic ordering of the binary strings $\mathbf{n}$, it proves convenient to regroup them in terms of the number of 1's they contain. We then introduce the enumeration of the $2^{N}$ binary $N$-tuples known as combinatorial numbering of degree $p$ [61], which we refer to as combinadic ordering for the sake of brevity. For fixed $p=0,1,2, \ldots, N$, one bijectively associates with each of the $\left(\begin{array}{l}N \\ p\end{array}\right) N$-tuples $\mathbf{n}$, with $n_{i_{\ell}}=1$, at sites $i_{1}<\cdots<i_{p}$, the integers

$$
1 \leq \mathcal{N}_{p}=1+\sum_{\ell=1}^{p}\left(\begin{array}{c}
i_{\ell}-1 \\
\ell
\end{array}\right) \leq\left(\begin{array}{l}
N \\
p
\end{array}\right),
$$

where the binomial coefficients $\left(\begin{array}{c}i_{\ell}-1 \\ \ell\end{array}\right)$ are set to vanish if $i_{\ell}-1<\ell$. According to such a numbering, we identify n with a unique $\mathcal{N}_{p}$ for some $p=0,1, \ldots, N$; then, the stationary state $\rho_{\infty}$ may be written as

$$
\rho_{\infty}=\sum_{p=0}^{N} \sum_{\mathcal{N}_{p}=1}^{\left(\begin{array}{l}
N \\
p
\end{array}\right)} \mathcal{L}_{\mathcal{N}_{p}}^{(p)}\left|\mathcal{N}_{p}\right\rangle\left\langle\mathcal{N}_{p}\right|
$$

where (see also Appendix G) the vectors $\left|\mathcal{N}_{p}\right\rangle$ now denote the fermionic eigenstates $b_{i_{1}}^{\dagger} \cdots b_{i_{p}}^{\dagger}|v\rangle$, while $\mathcal{L}_{\mathcal{N}_{p}}^{(p)}$ denotes the eigenvalues $\Lambda_{\mathbf{n}}$ in Eq. (54) corresponding to the binary $N$-tuples $\mathbf{n}$ with $p 1$ 's, indexed by the combinadic integer $\mathcal{N}_{p}$. Applications of the above formalism to the $N=2$ and $N=3$ cases can be found in Appendix F. Finally, in Appendix $\mathrm{H}$ it is shown that the stationary state $\rho_{\infty}$ has the 
following structure in terms of spin operators:

$$
\rho_{\infty}=\sum_{p=0}^{N} \sum_{\mathcal{N}_{p}^{\prime}, \mathcal{N}_{p}^{\prime \prime}} \mathcal{S}_{\mathcal{N}_{p}^{\prime} \mathcal{N}_{p}^{\prime \prime}}^{(p)} \prod_{\ell=1}^{N}\left(X_{n_{\ell}^{\prime}}^{(\ell)}\left(X_{n_{\ell}^{\prime \prime}}^{(\ell)}\right)^{\dagger}\right)
$$

where the expressions of the scalar quantities $\mathcal{S}_{\mathcal{N}_{p}^{\prime} \mathcal{N}_{p}^{\prime \prime}}^{(p)}$ are given in Eq. (G9) of Appendix G, while the singlespin operators read $X_{0}^{(\ell)}=\left[\left(1-\sigma_{z}^{(\ell)}\right) / 2\right]$ and $X_{1}^{(\ell)}=\sigma_{+}^{(\ell)}$, where $n_{\ell}^{\prime}$ and $n_{\ell}^{\prime \prime}$ are the digits of the binary $N$-tuples identified by the combinadic indices $\mathcal{N}_{p}^{\prime}$ and $\mathcal{N}_{p}^{\prime \prime}$. The above expression of the stationary state $\rho_{\infty}$ can thus be algorithmically computed for any $N$; the cases $N=2,3$ provide concrete and informative analytic instances of the above structure and are dealt with in Appendix I.

\section{B. Two-spin asymptotic density matrices}

The spin-operator expression of the stationary state $\rho_{\infty}$ is useful to investigate the entanglement content of any pair of spins along the chain and its dependence on their positions $N \geq s>r \geq 1$ and on temperature differences. We measure the two-spin entanglement by means of concurrence $[62,63]$. For a bipartite qubit system described by density matrix $\rho$, the concurrence is given by

$$
C(\rho)=\max \left\{0, \sqrt{\lambda_{1}}-\sqrt{\lambda_{2}}-\sqrt{\lambda_{3}}-\sqrt{\lambda_{4}}\right\}
$$

where $\lambda_{i}$ are the (positive) eigenvalues of the matrix $\rho\left(\sigma_{y} \otimes \sigma_{y}\right) \rho^{*}\left(\sigma_{y} \otimes \sigma_{y}\right)$, with $\rho^{*}$ the complex conjugate of $\rho$ in the basis in which $\sigma_{y}$ is the standard second Pauli matrix.

To quantify bipartite entanglement among two spins in the chain, we compute the concurrence of the two-spin reduced density matrix, which is obtained by tracing over the spins at sites different from $r$ and $s$. In Appendix J, it is shown that the asymptotic two-spin reduced density matrices obtained from the stationary state $\rho_{\infty}$ read

$$
\rho_{(r, s)}=\left(\begin{array}{cccc}
a & 0 & 0 & 0 \\
0 & b & c & 0 \\
0 & c & d & 0 \\
0 & 0 & 0 & e
\end{array}\right)
$$

with off-diagonal entry

$$
c=\sum_{p=0}^{N} \sum_{\substack{\mathcal{N}_{p}^{(r s)}(1,0) \\ \mathcal{N}_{p}^{(r s)}(0,1)}} \mathcal{S}_{\mathcal{N}_{p}^{(r s)}(1,0) \mathcal{N}_{p}^{(r s)}(0,1)}^{(p)}
$$

Here, the combinadic indices of the entries of $\mathcal{S}^{(p)}$ contributing to the only off-diagonal term $c$ involve different sites $r$ and $s$. The combinadic indices are, instead, the same for the entries of $\mathcal{S}^{(p)}$ contributing to the diagonal entries:

$$
\begin{aligned}
a= & \sum_{p=0}^{N} \sum_{\mathcal{N}_{p}^{(r s)}(1,1)} \mathcal{S}_{\mathcal{N}_{p}^{(r s)}(1,1) \mathcal{N}_{p}^{(r s)}(1,1)}^{(p)}, \\
b= & \sum_{p=0}^{N} \sum_{\mathcal{N}_{p}^{(r s)}(1,0)} \mathcal{S}_{\mathcal{N}_{p}^{(r s)}(1,0) \mathcal{N}_{p}^{(r s)}(1,0)}^{(p)}, \\
d= & \sum_{p=0}^{N} \sum_{\mathcal{N}_{p}^{(r s)}(0,1)} \mathcal{S}_{\mathcal{N}_{p}^{(r s)}(0,1) \mathcal{N}_{p}^{(r s)}(0,1)}^{(p)}, \\
e= & \sum_{p=0}^{N} \sum_{\mathcal{N}_{p}^{(r s)}(0,0)} \mathcal{S}_{\mathcal{N}_{p}^{(r s)}(0,0) \mathcal{N}_{p}^{(r s)}(0,0)}^{(p)}
\end{aligned}
$$

For such states, the concurrence takes the following analytic expression:

$$
C(r, s)=2 \max \{0,(|c|-\sqrt{a e})\},
$$

and hence the stationary bipartite entanglement corresponding to a nonvanishing positive $C(r, s)$ can be evaluated as a function of the sites $r$ and $s$ and their distance $s-r$. The concurrences $C(1,2), C(2,3)$, and $C(1,3)$ for a three-spin chain are studied in Appendix K 1.

\section{Two-spin concurrence}

In this section, we study the stationary two-spin entanglement in a $N$-spin chain. In doing so, we use Appendix K2, which shows how the coefficients $a, b, c$, $d$, and $e$ appearing in the concurrence $C(r, s)$ in Eq. (92) can be algorithmically reconstructed. The quantity $C(r, s)$ depends on the parameters $\Delta$ and $g$ of the chain Hamiltonian, on the temperatures $T_{L, R}$, on the number of spins, $N$, and on the spin sites $0 \leq r \leq s \leq N$.

First, although the algorithm developed in Appendix K 2 works for all $N$, its algorithmic implementation rapidly becomes time-consuming so that, in the following figures, we focus upon a chain consisting of $N=8$ spins. In full generality, we observe that, similarly to the sink and source terms in Eqs. (73) and (74), the bipartite entanglement between any pair of sites scales as $1 / N$; this follows from the fact that, for large $N$, such is the leading order of the matrix elements $\mathcal{S}_{\mathcal{N}_{p}^{\prime} \mathcal{N}_{p}^{\prime \prime}}^{(2)}$ in Eq. (G9). In turn, such a behavior is due to the fact that the transition frequencies $\omega_{\ell}$ in Eq. (45), and thus the eigenvalues in Eq. (54), are of order 1 with respect to $N$, while the quantities $\mathcal{D}_{\mathcal{N}_{p}^{\prime} \mathcal{N}_{p}^{\prime \prime}}^{(p)}$ introduced in Remark 5 are of order $1 /(\sqrt{N})^{p}$ and, in each of the expressions given in Eq. (87)-(91), there appear sums from $p=1$ to $p=N$ of products of pairs of such terms. 


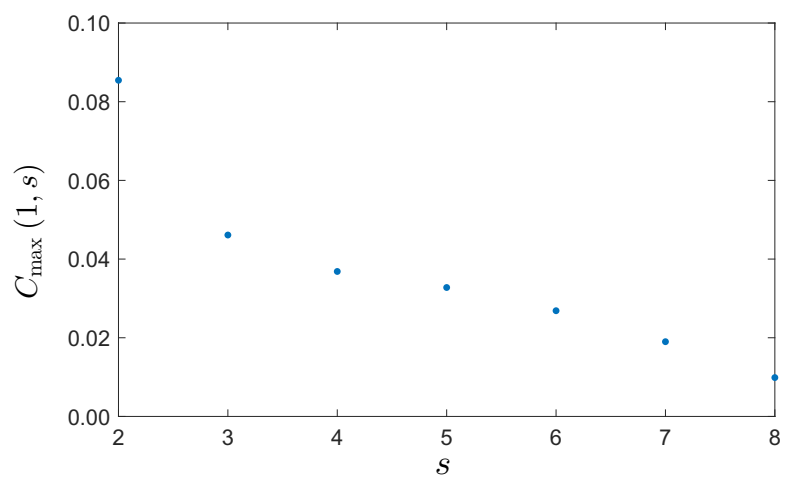

FIG. 3. The maximum achievable entanglement between sites 1 and $s=2,3, \ldots 8$ by varying $T_{R}$. Here, $N=8, \lambda=1, T_{L}=$ $0, \Delta=15$, and $g=7.8$ is close to its upper bound in Eq. (53).

Second, just as in the case of source and sink terms and of heat flows, we set $T_{L}=0$ and then inspect the dependence on the right temperature $T_{R}$ only. What one expects by letting $T_{L}>0$ is that when $T_{R}=T_{L}>0$, one reaches the Gibbs state in Eq. (62). This thermal-equilibrium state cannot provide transport effects, for $n_{L}\left(\omega_{\ell}\right)=n_{R}\left(\omega_{\ell}\right)$, but may, however, support bipartite entanglement at finite nonvanishing temperatures. On the other hand, for $T_{L}=T_{R}=$ 0 , the state becomes the vacuum state $|v\rangle$ in Eq. (25), which is clearly separable.

The expected features of the concurrence $C(r, s)$ are that by increasing the distance $s-r$ between the spins with fixed $r$, the maximum achievable bipartite entanglement $C_{\max }(r, s)$ diminishes, as shown in Fig. 3, while the concurrence itself vanishes at lower temperatures, in agreement with the fact that distance and temperature play against correlations. Furthermore, the lack of translational invariance makes $C(r, s)$ depend not only on $s-r$ but also on the position $r$ of the first spin.

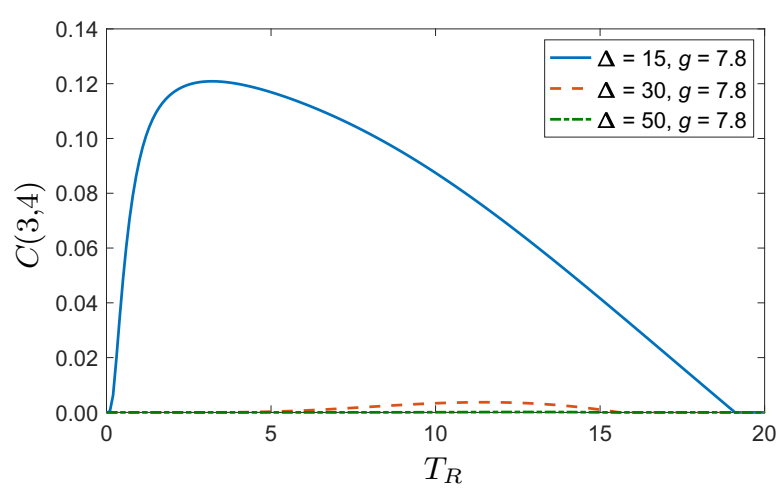

FIG. 4. The bipartite entanglement between spins 3 and 4 , as measured by the concurrence $C(3,4)$, versus $T_{R}$ for $N=8, T_{L}=$ 0 , with $\Delta=15,30$, and 50 and $g$ close to the saturation value relative to $\Delta=15$.

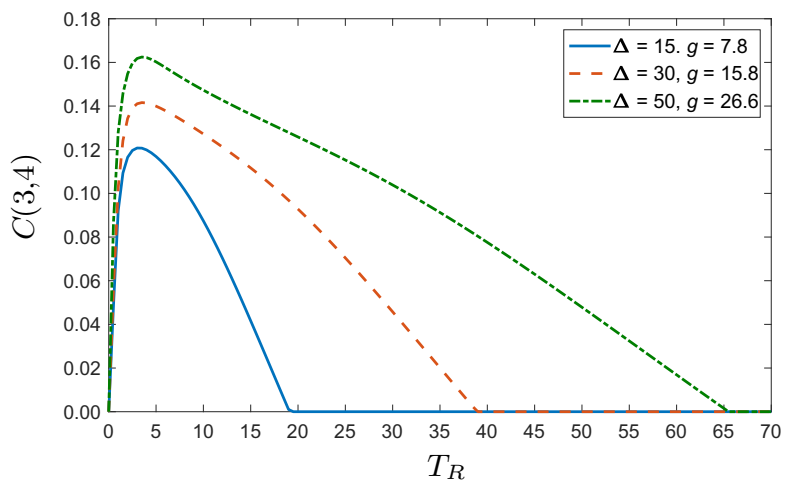

FIG. 5. The bipartite entanglement between spins 3 and 4 , as measured by the concurrence $C(3,4)$, versus $T_{R}$ for $N=8, T_{L}=$ 0 , with $\Delta=15,30$, and 50 and $g$ close to the corresponding saturation values.

As regards the dependence of the concurrence on the parameters $\Delta$ and $g$, Fig. 4 first shows that, with temperature $T_{L}=0$, and $g$ fixed, close to the upper bound of Eq. (53) for $\Delta=15$, the entanglement as a function of $T_{R}$ diminishes while increasing $\Delta$.

This behavior agrees with the fact that by augmenting the transverse external field, the spins all tend to become parallel and thus the stationary state becomes separable. On the other hand, by increasing $g$, the spins interact more strongly, thus favoring the generation of quantum correlations that may persist asymptotically against temperature. In fact, the further away $g$ is from the saturation value at given $\Delta$, the smaller is the achieved entanglement, the larger is the temperature at which it appears, and the smaller is the temperature at which it disappears. Specifically, the chosen value of $g$ is sufficient to generate entanglement for $\Delta=15,30$, but not for $\Delta=50$, while increasing $g$ beyond the saturation value for $\Delta=15$ would violate the condition assumed throughout the paper, namely, that the transition frequencies $\omega_{\ell}$ in Eq. (45) are positive.

Instead, in Fig. 5, under the same conditions as in Fig. 4, the values of the interaction strength $g$ are chosen close to the saturation bound given in Eq. (53) for each $\Delta$. The graph shows that in this case the highest possible $g$, despite the higher values of $\Delta$, contributes to the creation of entanglement as soon as $T_{R}>0$; moreover, it also makes it last up to higher values of $T_{R}$.

\section{DISCUSSION}

Spin chains coupled to external baths at their endpoints represent paradigmatic models for the study of transport properties in quantum many-body systems, as they allow the precise analysis of the behavior of spin and heat flows along the chain. So far, analytic treatments of the asymptotic transport properties in these systems have been obtained assuming only ad hoc couplings between 
the system and the external baths, those that allow the chain steady state to be expressed in terms of the so-called "matrix product states" [20].

Here, instead taking an energy-preserving coupling to the end baths, with strength allowing the weak-coupling limit, we are able to derive an exact analytic expression for the unique steady state of a generic $N$-site spin-1/2 chain, with $X X$-type interspin interaction, in a transverse constant magnetic field. This allows us to discuss in detail the open transport properties of the model, treated in the so-called global approach, revealing the presence of sink and source terms in the spin flow continuity equation that have never been pointed out before (except in the $N=3$ case [50]).

In addition, having the explicit form of the system asymptotic stationary state allows us to analyze the entanglement properties of the chain. In particular, a procedure is devised that is able to algorithmically provide the explicit expression of the reduced two-spin density matrix for any two sites along the chain. The behavior of the corresponding entanglement content of the reduced state, as measured by concurrence, is discussed in some relevant cases in terms of the parameters of the system Hamiltonian and of the bath temperatures. While increasing the bath temperatures and the magnitude of the external magnetic field counteracts entanglement, sufficiently high values of the interspin interaction coupling constant would always allow the presence of asymptotic entanglement among any couple of close enough sites.

In addition, these results show that, for generic $N$, there is no apparent relation between the behavior of heat flow and two-site entanglement as a function of the bath temperatures, as claimed in the literature for the special case $N=2$ [64]: entanglement in the chain is generated independently from the heat flow and even in the absence of it, as in the case of isothermal baths. Furthermore, the two quantities behave rather differently with respect to the length of the chain: while the concurrence vanishes as $1 / N$, the heat flow does not.

We are confident that these findings will stimulate further research on the use of many-body systems, and spin chains in particular, for modeling quantum transport processes, in view of possible applications in quantum technology.

\section{ACKNOWLEDGMENTS}

F.B. and R.F. acknowledge that their research has been conducted within the framework of the Trieste Institute for Theoretical Quantum Technologies. L.M. acknowledges financial support by the Sharif University of Technology, Office of the Vice President for Research, under Grant No. G930209 and hospitality by the Abdus Salam International Centre for Theoretical Physics (ICTP), where parts of this work were completed.
The authors contributed equally to this study and are listed in alphabetical order.

\section{APPENDIX A: DIAGONALIZATION OF THE SPIN-CHAIN HAMILTONIAN}

With $B_{N}$ the $N \times N$ tridiagonal symmetric matrix with entries $\left(B_{N}\right)_{i j}=\delta_{j, i+1}$ for $0 \leq i \leq j \leq N$, the Hamiltonian

$$
\widetilde{H}=\gamma \sum_{j=1}^{N} a_{j}^{\dagger} a_{j}+\sum_{j=1}^{N-1}\left(a_{j}^{\dagger} a_{j+1}+a_{j+1}^{\dagger} a_{j}\right)
$$

reads $\widetilde{H}=\sum_{j, k=1}^{N} h_{j k} a_{j}^{\dagger} a_{k}$ where $h=\gamma \mathbb{I}+B_{N}$. Since

$$
\operatorname{det}\left(x-B_{N+2}\right)=x \operatorname{det}\left(x-B_{N+1}\right)-\operatorname{det}\left(x-B_{N}\right),
$$

the same equation is satisfied by the associated characteristic polynomial $p_{N}(x)$ :

$$
p_{N+2}(x)-x p_{N+1}(x)+p_{N}(x)=0 .
$$

Setting $x=2 \cos \theta$, one finds the solution

$$
p_{N}(x)=C_{1} e^{i N \theta}+C_{2} e^{-i N \theta} .
$$

From $p_{1}(x)=x$ and $p_{2}(x)=x^{2}-1$, one fixes the coefficients

$$
C_{1}=\frac{e^{i \theta}}{e^{i \theta}-e^{-i \theta}}, \quad C_{2}=-\frac{e^{-i \theta}}{e^{i \theta}-e^{-i \theta}},
$$

and hence $p_{N}(x)=\sin ((N+1) \theta) / \sin \theta$.

Since for $\theta=0[\theta=\pi], p_{N}(2)=N+1\left[p_{N}(-2)=\right.$ $\left.(-)^{N}(N+1)\right]$, the only zeroes of $p_{N}(x)$ are at $\theta_{k}=$ $[k \pi /(N+1)], k=1,2, \ldots, N$. It thus follows that the eigenvalues of $h=\gamma \mathbb{I}+B$ are

$$
\lambda_{k}=\gamma+2 \cos \left(\frac{k \pi}{N+1}\right) .
$$

Finally, one can check that the symmetric matrix

$$
U=\left[u_{j k}\right]_{j, k=1}^{N}, \quad u_{j k}=\sqrt{\frac{2}{N+1}} \sin \left(\frac{j k \pi}{N+1}\right) .
$$

is also orthogonal. It can be directly checked that

$$
U B U=\operatorname{diag}\left[2 \cos \left(\frac{\ell \pi}{N+1}\right)\right]_{\ell=1}^{N}
$$


APPENDIX B: ENERGIES AND EIGENVECTORS OF TWO- AND THREE-SPIN CHAINS

\section{Two-spin chain}

For a chain consisting of only two spins, as in Refs. [60, 64-66], $N=2$ and Eq. (35) yield the energies

$$
E_{00}=-2 \Delta, \quad E_{10}=2 g, \quad E_{01}=-2 g, \quad E_{11}=2 \Delta .
$$

Furthermore, from Eq. (A6), the unitary matrix $U$ results:

$$
U=\frac{1}{\sqrt{2}}\left(\begin{array}{cc}
1 & 1 \\
1 & -1
\end{array}\right) \text {. }
$$

Therefore, applying Eq. (37), one obtains

$$
\begin{aligned}
b_{1} & =\frac{\sigma_{-}^{(1)}-\sigma_{z}^{(1)} \sigma_{-}^{(2)}}{\sqrt{2}}, \quad b_{2}=\frac{\sigma_{-}^{(1)}+\sigma_{z}^{(1)} \sigma_{-}^{(2)}}{\sqrt{2}}, \\
b_{1} b_{2} & =-\sigma_{-}^{(1)} \sigma_{-}^{(2)}
\end{aligned}
$$

so that using Eqs. (31) and (25), one can recast the eigenvectors $\left|n_{1} n_{2}\right\rangle$ relative to the eigenvalues $E_{n_{1} n_{2}}$ using the standard basis, $|\uparrow\rangle,|\downarrow\rangle$. Indeed, $|v\rangle=|00\rangle=|\downarrow \downarrow\rangle$, and hence

$$
\begin{aligned}
& |00\rangle=|\downarrow \downarrow\rangle, \quad|10\rangle=\frac{|\uparrow \downarrow\rangle+|\downarrow \uparrow\rangle}{\sqrt{2}} \\
& |01\rangle=\frac{|\uparrow \downarrow\rangle-|\downarrow \uparrow\rangle}{\sqrt{2}}, \quad|11\rangle=|\uparrow \uparrow\rangle .
\end{aligned}
$$

\section{Three-spin chain}

In the case of a three-spin chains [50], setting $N=3$, the eigenvalues of the Hamiltonian given in Eq. (1) are

$$
E_{n_{1} n_{2} n_{3}}=\Delta\left(2 \sum_{\ell=1}^{3} n_{\ell}-3\right)+4 g \sum_{\ell=1}^{3} n_{\ell} \cos \left(\frac{\ell \pi}{4}\right) .
$$

Explicitly, they and their corresponding eigenvectors read as follows:

$$
\begin{aligned}
& |000\rangle=|v\rangle, \quad E_{000}=-3 \Delta, \\
& |100\rangle=b_{1}^{\dagger}|000\rangle, \quad E_{100}=-\Delta+2 \sqrt{2} g, \\
& |010\rangle=b_{2}^{\dagger}|000\rangle, \quad E_{010}=-\Delta, \\
& |001\rangle=b_{3}^{\dagger}|000\rangle, \quad E_{001}=-\Delta-2 \sqrt{2} g, \\
& |110\rangle=b_{1}^{\dagger} b_{2}^{\dagger}|000\rangle, \quad E_{110}=\Delta+2 \sqrt{2} g, \\
& |101\rangle=b_{1}^{\dagger} b_{3}^{\dagger}|000\rangle, \quad E_{101}=\Delta, \\
& |011\rangle=b_{2}^{\dagger} b_{3}^{\dagger}|000\rangle, \quad E_{011}=\Delta-2 \sqrt{2} g \\
& |111\rangle=b_{1}^{\dagger} b_{2}^{\dagger} b_{3}^{\dagger}|000\rangle, \quad E_{111}=3 \Delta .
\end{aligned}
$$

The correspondence with the eigenvalues $E_{j}$ in Ref. [50] is as follows:

$$
\begin{array}{llll}
E_{000}=E_{2}, & E_{100}=E_{7}, & E_{010}=E_{4}, & E_{001}=E_{6}, \\
E_{110}=E_{5}, & E_{101}=E_{3}, & E_{011}=E_{8}, & E_{111}=E_{1} .
\end{array}
$$

Since the $3 \times 3$ matrix $U$ in Eq. (A6) reads

$$
U=\frac{1}{2}\left(\begin{array}{ccc}
1 & \sqrt{2} & 1 \\
\sqrt{2} & 0 & -\sqrt{2} \\
1 & -\sqrt{2} & 1
\end{array}\right)
$$

then $\sigma_{ \pm} \sigma_{z}=\mp \sigma_{ \pm}$and Eq. (38) yield

$$
\begin{aligned}
b_{1}^{\dagger} & =\frac{1}{2}\left(\sigma_{+}^{(1)}-\sqrt{2} \sigma_{z}^{(1)} \sigma_{+}^{(2)}+\sigma_{z}^{(1)} \sigma_{z}^{(2)} \sigma_{+}^{(3)}\right), \\
b_{2}^{\dagger} & =\frac{1}{\sqrt{2}}\left(\sigma_{+}^{(1)}-\sigma_{z}^{(1)} \sigma_{z}^{(2)} \sigma_{+}^{(3)}\right), \\
b_{3}^{\dagger} & =\frac{1}{2}\left(\sigma_{+}^{(1)}+\sqrt{2} \sigma_{z}^{(1)} \sigma_{+}^{(2)}+\sigma_{z}^{(1)} \sigma_{z}^{(2)} \sigma_{+}^{(3)}\right), \\
b_{1}^{\dagger} b_{2}^{\dagger} & =-\frac{1}{2}\left(\sigma_{+}^{(1)} \sigma_{+}^{(2)}-\sqrt{2} \sigma_{+}^{(1)} \sigma_{z}^{(2)} \sigma_{+}^{(3)}+\sigma_{+}^{(2)} \sigma_{+}^{(3)}\right), \\
b_{2}^{\dagger} b_{3}^{\dagger} & =-\frac{1}{2}\left(\sigma_{+}^{(1)} \sigma_{+}^{(2)}+\sqrt{2} \sigma_{+}^{(1)} \sigma_{z}^{(2)} \sigma_{+}^{(3)}+\sigma_{+}^{(2)} \sigma_{+}^{(3)}\right), \\
b_{1}^{\dagger} b_{3}^{\dagger} & =-\frac{1}{\sqrt{2}}\left(\sigma_{+}^{(1)} \sigma_{+}^{(2)}-\sigma_{+}^{(2)} \sigma_{+}^{(3)}\right), \\
b_{1}^{\dagger} b_{2}^{\dagger} b_{3}^{\dagger} & =-\sigma_{+}^{(1)} \sigma_{+}^{(2)} \sigma_{+}^{(3)} .
\end{aligned}
$$

The expressions of the eigenvectors $\left|n_{1} n_{2} n_{3}\right\rangle$ in the spin standard basis and their correspondence with the eigenvectors obtained in Ref. [50] are reported in Appendix C 2.

\section{APPENDIX C: LINDBLAD OPERATORS FOR TWO- AND THREE-SPIN CHAINS}

\section{Two-spin chain}

In the case of $N=2$, from Eq. (45) one computes the following transition frequencies:

$$
\omega_{1}=2(\Delta+g), \quad \omega_{2}=2(\Delta-g) .
$$

Using Eqs. (46) and (50), the following Lindblad operators ensue for the open two-spin chain:

$$
\begin{aligned}
A_{L}^{\dagger}\left(\omega_{1}\right) & =\frac{|10\rangle\langle 00|+| 11\rangle\langle 01|}{\sqrt{2}}, \\
A_{L}^{\dagger}\left(\omega_{2}\right) & =\frac{|01\rangle\langle 00|-| 11\rangle\langle 10|}{\sqrt{2}},
\end{aligned}
$$




$$
\begin{aligned}
A_{R}^{\dagger}\left(\omega_{1}\right) & =\frac{|10\rangle\langle 00|-| 11\rangle\langle 0|}{\sqrt{2}}, \\
A_{R}^{\dagger}\left(\omega_{2}\right) & =-\frac{|01\rangle\langle 00|+| 11\rangle\langle 10|}{\sqrt{2}} .
\end{aligned}
$$

According to the discussion before Remark 4, in order to have all of them contribute to dissipation, one must set $g \leq \Delta$.

\section{Three-spin chain}

Setting $N=3$, from Eq. (45) we obtain the three frequencies

$$
\omega_{1}=2(\Delta+\sqrt{2} g), \quad \omega_{2}=2 \Delta, \quad \omega_{3}=2(\Delta-\sqrt{2} g),
$$

which correspond to the three frequencies $\omega_{1}, \omega_{0}$, and $\omega_{2}$ in Ref. [50]. Furthermore, Eqs. (46) and (50) yield the leftbath Lindblad operators

$$
\begin{aligned}
A_{L}^{\dagger}\left(\omega_{1}\right)= & \frac{1}{2}(|100\rangle\langle 000|+| 110\rangle\langle 010| \\
& +|101\rangle\langle 001|+| 111\rangle\langle 011|), \\
A_{L}^{\dagger}\left(\omega_{2}\right)= & \frac{1}{\sqrt{2}}(|010\rangle\langle 000|-| 110\rangle\langle 100| \\
& -|111\rangle\langle 101|+| 011\rangle\langle 001|), \\
A_{L}^{\dagger}\left(\omega_{3}\right)= & \frac{1}{2}(|001\rangle\langle 000|-| 101\rangle\langle 100| \\
& -|011\rangle\langle 010|+| 111\rangle\langle 110|),
\end{aligned}
$$

and the right-bath Lindblad operators

$$
\begin{aligned}
A_{R}^{\dagger}\left(\omega_{1}\right)= & \frac{1}{2}(|011\rangle\langle 001|+| 111\rangle\langle 101| \\
& -|010\rangle\langle 000|-| 110\rangle\langle 100|), \\
A_{R}^{\dagger}\left(\omega_{2}\right)= & \frac{1}{\sqrt{2}}(|011\rangle\langle 001|+| 111\rangle\langle 101| \\
& -|010\rangle\langle 000|-| 110\rangle\langle 100|), \\
A_{R}^{\dagger}\left(\omega_{3}\right)= & \frac{1}{2}(|001\rangle\langle 000|+| 101\rangle\langle 100| \\
& +|011\rangle\langle 010|+| 111\rangle\langle 110|) .
\end{aligned}
$$

According to the discussion before Remark 4, in order to have all of them contribute to dissipation, one must set $g \leq$ $(\Delta / \sqrt{2})$.
In terms of the eigenstates $|\uparrow\rangle$ and $|\downarrow\rangle$ of $\sigma_{z}$, using the fact that $|v\rangle=|000\rangle=|\downarrow \downarrow \downarrow\rangle$, one can reexpress the eigenvectors $\left|n_{1} n_{2} n_{3}\right\rangle$ in the spin standard basis:

$$
|000\rangle=|\downarrow \downarrow \downarrow\rangle=\left|E_{2}\right\rangle,
$$

in the case of zero excitations, while for one excitation,

$$
\begin{aligned}
|100\rangle & =b_{1}^{\dagger}|000\rangle=\frac{1}{2}(|\uparrow \downarrow \downarrow\rangle+\sqrt{2}|\downarrow \uparrow \downarrow\rangle+|\downarrow \downarrow \uparrow\rangle) \\
& =\left|E_{7}\right\rangle, \quad(\mathrm{C} 14) \\
|010\rangle & =b_{2}^{\dagger}|000\rangle=\frac{1}{\sqrt{2}}(|\uparrow \downarrow \downarrow\rangle-|\downarrow \downarrow \uparrow\rangle)=\left|E_{4}\right\rangle,
\end{aligned}
$$

$$
\begin{aligned}
|001\rangle & =b_{3}^{\dagger}|000\rangle=\frac{1}{2}(|\uparrow \downarrow \downarrow\rangle-\sqrt{2}|\downarrow \uparrow \downarrow\rangle+|\downarrow \downarrow \uparrow\rangle) \\
& =\left|E_{6}\right\rangle,
\end{aligned}
$$

and for two excitations,

$$
\begin{aligned}
|110\rangle & =b_{1}^{\dagger} b_{2}^{\dagger}|000\rangle=-\frac{1}{2}(|\uparrow \uparrow \downarrow\rangle+\sqrt{2}|\uparrow \downarrow \uparrow\rangle+|\downarrow \uparrow \uparrow\rangle) \\
& =-\left|E_{5}\right\rangle, \\
|101\rangle & =b_{1}^{\dagger} b_{3}^{\dagger}|000\rangle=-\frac{1}{\sqrt{2}}(|\uparrow \uparrow \downarrow\rangle-|\downarrow \uparrow \uparrow\rangle) \\
& =-\left|E_{3}\right\rangle, \\
|011\rangle & =b_{2}^{\dagger} b_{3}^{\dagger}|000\rangle=-\frac{1}{2}(|\uparrow \uparrow \downarrow\rangle-\sqrt{2}|\uparrow \downarrow \uparrow\rangle+|\downarrow \uparrow \uparrow\rangle) \\
& =-\left|E_{8}\right\rangle .
\end{aligned}
$$

Finally, in the case of three excitations, one finds

$$
|111\rangle=b_{1}^{\dagger} b_{2}^{\dagger} b_{3}^{\dagger}|000\rangle=-|\uparrow \uparrow \uparrow\rangle=-\left|E_{1}\right\rangle,,
$$

the difference in the overall sign depending on the chosen ordering of the creation operators $b_{\ell}^{\dagger}$. The correspondence with the $\left|E_{k}\right\rangle$ obtained in Ref. [50] is confirmed after noting that there $|0\rangle=|\uparrow\rangle$ and $|1\rangle=|\downarrow\rangle$.

In the same vein, using Eqs. (C13)-(C20), one recasts the Lindblad operators in Eqs. (C7)-(C12) as

$$
\begin{array}{r}
A_{L}^{\dagger}\left(\omega_{1}\right)=\frac{1}{2}\left(\left|E_{7}\right\rangle\left\langle E_{2}|-| E_{5}\right\rangle\left\langle E_{4}|-| E_{3}\right\rangle\left\langle E_{6}|+| E_{1}\right\rangle\left\langle E_{8}\right|\right), \\
(\mathrm{C} 21) \\
A_{L}^{\dagger}\left(\omega_{2}\right)=\frac{1}{\sqrt{2}}\left(\left|E_{4}\right\rangle\left\langle E_{2}|+| E_{5}\right\rangle\left\langle E_{7}|-| E_{1}\right\rangle\left\langle E_{3}|-| E_{8}\right\rangle\left\langle E_{6}\right|\right),
\end{array}
$$

$A_{L}^{\dagger}\left(\omega_{3}\right)=\frac{1}{2}\left(\left|E_{6}\right\rangle\left\langle E_{2}|+| E_{3}\right\rangle\left\langle E_{7}|+| E_{8}\right\rangle\left\langle E_{4}|+| E_{1}\right\rangle\left\langle E_{5}\right|\right)$, 
and

$$
\begin{array}{r}
A_{R}^{\dagger}\left(\omega_{1}\right)=\frac{1}{2}\left(\left|E_{7}\right\rangle\left\langle E_{2}|+| E_{5}\right\rangle\left\langle E_{4}|+| E_{3}\right\rangle\left\langle E_{6}|+| E_{1}\right\rangle\left\langle E_{8}\right|\right), \\
(\mathrm{C} 24) \\
A_{R}^{\dagger}\left(\omega_{2}\right)=\frac{1}{\sqrt{2}}\left(\left|E_{5}\right\rangle\left\langle E_{7}|-| E_{4}\right\rangle\left\langle E_{2}|-| E_{8}\right\rangle\left\langle E_{6}|+| E_{1}\right\rangle\left\langle E_{3}\right|\right), \\
A_{R}^{\dagger}\left(\omega_{3}\right)=\frac{1}{2}\left(\left|E_{6}\right\rangle\left\langle E_{2}|+| E_{1}\right\rangle\left\langle E_{5}|-| E_{3}\right\rangle\left\langle E_{7}|-| E_{8}\right\rangle\left\langle E_{4}\right|\right) .
\end{array}
$$

These expressions coincide with those found in Ref. [50].

\section{APPENDIX D: STATIONARY STATE}

\section{Uniqueness of the stationary state}

Consider the commutator of a generic chain operator $X=\sum_{\mathbf{p}, \mathbf{q}} X_{\mathbf{p q}}|\mathbf{p}\rangle\langle\mathbf{q}|$, in the energy eigenbasis given in Eq. (34), with the Lindblad operator given in Eq. (46), and set it equal to zero:

$$
\begin{aligned}
\left\langle\mathbf{r}\left|\left[X, A_{L}^{\dagger}\left(\omega_{\ell}\right)\right]\right| \mathbf{s}\right\rangle= & u_{1 \ell} \sum_{\mathbf{p}, \mathbf{q}, \widehat{\mathbf{n}}_{\ell}}(-1)^{\sum_{j=1}^{\ell-1} n_{j}} X_{\mathbf{p q}} \\
& \times\left\langle\mathbf{r}\left|\left[|\mathbf{p}\rangle\left\langle\mathbf{q}|,| \mathbf{n}_{1_{\ell}}\right\rangle\left\langle\mathbf{n}_{0_{\ell}}\right|\right]\right| \mathbf{s}\right\rangle=0 .
\end{aligned}
$$

This yields

$$
X_{\mathbf{r s}_{1_{\ell}}}(-1)^{\sum_{j=1}^{\ell-1} s_{j}} \delta_{s_{\ell} 0}=X_{\mathbf{r}_{0_{\ell}} \mathbf{s}}(-1)^{\sum_{j=1}^{\ell-1} r_{j}} \delta_{r_{\ell} 1},
$$

and hence choosing $r_{\ell}=0$ and $s_{\ell}=0$ gives $X_{\mathbf{r}_{\ell}} \mathbf{s}_{1_{\ell}}=0$. Changing $A_{L}^{\dagger}\left(\omega_{\ell}\right)$ into $A_{R}^{\dagger}\left(\omega_{\ell}\right)$ yields $X_{\mathbf{r}_{1_{\ell}} \mathbf{s}_{0_{\ell}}}=0$; thus, the only nonvanishing $X$ commuting with all Lindblad operators must be diagonal in the energy eigenbasis: namely, $X=\sum_{\mathbf{n}} X_{\mathbf{n n}}|\mathbf{n}\rangle\langle\mathbf{n}|$. On the other hand, choosing $r_{\ell}=1$ and $s_{\ell}=0$, Eq. (D1) yields $X_{\mathbf{n}_{\ell} \mathbf{n}_{1_{\ell}}}=X_{\mathbf{n}_{0_{\ell}} \mathbf{n}_{0_{\ell}}}$ for all $\ell=$ $1,2, \ldots, N$ and hence $X$ must be a multiple of the identity.

\section{Structure of the stationary state}

Inserting the eigenprojections $P_{\mathbf{k}}$ into Eqs. (13) and (14), one obtains

$$
\begin{aligned}
& \mathbb{D}_{\omega_{\ell}}^{(L)}\left[P_{\mathbf{k}}\right]=u_{1 \ell}^{2}\left(C_{\omega_{\ell}}^{(L)} \delta_{k_{\ell} 1}-\widetilde{C}_{\omega_{\ell}}^{(L)} \delta_{k_{\ell} 0}\right)\left(P_{\mathbf{k}_{0_{\ell}}}-P_{\mathbf{k}_{1_{\ell}}}\right), \\
& \mathbb{D}_{\omega_{\ell}}^{(R)}\left[P_{\mathbf{k}}\right]=u_{N \ell}^{2}\left(C_{\omega_{\ell}}^{(R)} \delta_{k_{\ell} 1}-\widetilde{C}_{\omega_{\ell}}^{(R)} \delta_{k_{\ell} 0}\right)\left(P_{\mathbf{k}_{0_{\ell}}}-P_{\mathbf{k}_{\mathbf{k}_{\ell}}}\right) .
\end{aligned}
$$

From Eq. (12), using the two previous expressions, one finds that

$$
\mathbb{D}\left[P_{\mathbf{k}}\right]=\lambda^{2} \sum_{\ell=1}^{N}\left(\delta_{k_{\ell} 1} d_{\ell}-\delta_{k_{\ell} 0} \widetilde{d}_{\ell}\right)\left(P_{\mathbf{k}_{0_{\ell}}}-P_{\mathbf{k}_{1_{\ell}}}\right),
$$

where, using Eqs. (15) and (16),

$$
\begin{aligned}
d_{\ell}= & C_{\omega_{\ell}}^{(L)} u_{1 \ell}^{2}+C_{\omega_{\ell}}^{(R)} u_{N \ell}^{2} \\
= & \frac{4 \pi}{N+1} \sin ^{2}\left(\frac{\pi \ell}{N+1}\right)\left[\left[h_{L}\left(\omega_{\ell}\right)\right]^{2}\left(1+n_{L}\left(\omega_{\ell}\right)\right)\right. \\
& \left.+\left[h_{R}\left(\omega_{\ell}\right)\right]^{2}\left(1+n_{R}\left(\omega_{\ell}\right)\right)\right], \\
\widetilde{d}_{\ell}= & \widetilde{C}_{\omega_{\ell}}^{(L)} u_{1 \ell}^{2}+\widetilde{C}_{\omega_{\ell}}^{(R)} u_{N \ell}^{2} \\
= & \frac{4 \pi}{N+1} \sin ^{2}\left(\frac{\pi \ell}{N+1}\right)\left[\left[h_{L}\left(\omega_{\ell}\right)\right]^{2} n_{L}\left(\omega_{\ell}\right)\right. \\
& \left.+\left[h_{R}\left(\omega_{\ell}\right)\right]^{2} n_{R}\left(\omega_{\ell}\right)\right] .
\end{aligned}
$$

Consider the diagonal expression $X_{\text {diag }}=\sum_{\mathbf{n}} x_{\mathbf{n}} P_{\mathbf{n}}$; then, the dissipator maps it into

$$
\mathbb{D}\left[X_{\text {diag }}\right]=\sum_{\mathbf{n}} \widetilde{x}_{\mathbf{n}} P_{\mathbf{n}}
$$

with

$$
\tilde{x}_{\mathbf{n}}=\operatorname{Tr}\left(P_{\mathbf{n}} \mathbb{D}\left[X_{\text {diag }}\right]\right)=\sum_{\mathbf{m}} x_{\mathbf{m}} \operatorname{Tr}\left(P_{\mathbf{n}} \mathbb{D}\left[P_{\mathbf{m}}\right]\right) .
$$

From Eq. (D4), it follows that

$$
\begin{aligned}
\mathbb{D}\left[X_{\text {diag }}\right] & =\lambda^{2} \sum_{\mathbf{n}} x_{\mathbf{n}} \sum_{\ell=1}^{N}\left(\delta_{n_{\ell} 1} d_{\ell}-\delta_{n_{\ell} 0} \widetilde{d}_{\ell}\right)\left(P_{\mathbf{n}_{0_{\ell}}}-P_{\mathbf{n}_{\mathbf{n}_{\ell}}}\right) \\
& =\sum_{\ell=1}^{N} \sum_{\hat{\mathbf{n}}_{\ell}}\left(d_{\ell} x_{\mathbf{n}_{1_{\ell}}}-\widetilde{d}_{\ell} x_{\mathbf{n}_{0_{\ell}}}\right)\left(P_{\mathbf{n}_{0_{\ell}}}-P_{\mathbf{n}_{1_{\ell}}}\right),
\end{aligned}
$$

and hence

$$
\tilde{x}_{\mathbf{n}}=\lambda^{2} \sum_{\ell=1}^{N}\left(\delta_{n_{\ell} 0}-\delta_{n_{\ell} 1}\right)\left(d_{\ell} x_{\mathbf{n}_{1_{\ell}}}-\tilde{d}_{\ell} x_{\mathbf{n}_{0_{\ell}}}\right) .
$$

Therefore, $\mathbb{D}\left[X_{\text {diag }}\right]=0$ is obtained by the factorized expressions

$$
x_{\mathbf{n}}=\prod_{\ell=1}^{N} x_{n_{\ell}}^{(\ell)}, \quad x_{0}^{(\ell)}=d_{\ell}, \quad x_{1}^{(\ell)}=\tilde{d}_{\ell} .
$$

All $x^{(\ell)} \geq 0$ and, after normalization, the uniqueness of the stationary state together with the expressions Eqs. (D5) and (D6) yield Eqs. (54)-(57). 


\section{APPENDIX E: SINK AND SOURCE CONTRIBUTIONS TO THE STATIONARY TRANSPORT PROPERTIES}

Given the stationary state in Eq. (54), in order to compute

$$
\mathfrak{Q}_{L}^{(k)}=\lambda^{2} \sum_{\ell=1}^{N} \operatorname{Tr}\left(\rho_{\infty} \widetilde{\mathbb{D}}_{\omega_{\ell}}^{(L)}\left[\sigma_{z}^{(k)}\right]\right)
$$

in Eq. (73), we need to evaluate mean values of the form

$$
\begin{aligned}
\left\langle\mathbf{n}\left|\tilde{\mathbb{D}}_{\omega_{\ell}}^{(L)}\left[\sigma_{z}^{(k)}\right]\right| \mathbf{n}\right\rangle & =C_{\omega_{\ell}}^{(L)}\left(\left\langle\mathbf{n}\left|A_{L}^{\dagger}\left(\omega_{\ell}\right) \sigma_{z}^{(k)} A_{L}\left(\omega_{\ell}\right)\right| \mathbf{n}\right\rangle\right. \\
& \left.-\frac{1}{2}\left\langle\mathbf{n}\left|\left\{A_{L}^{\dagger}\left(\omega_{\ell}\right) A_{L}\left(\omega_{\ell}\right), \sigma_{z}^{(k)}\right\}\right| \mathbf{n}\right\rangle\right) \\
& +\widetilde{C}_{\omega_{\ell}}^{(L)}\left(\left\langle\mathbf{n}\left|A_{L}\left(\omega_{\ell}\right) \sigma_{z}^{(k)} A_{L}^{\dagger}\left(\omega_{\ell}\right)\right| \mathbf{n}\right\rangle\right. \\
& \left.-\frac{1}{2}\left\langle\mathbf{n}\left|\left\{A_{L}\left(\omega_{\ell}\right) A_{L}^{\dagger}\left(\omega_{\ell}\right), \sigma_{z}^{(k)}\right\}\right| \mathbf{n}\right\rangle\right) .
\end{aligned}
$$

Using the expressions given in Eqs. (46)-(48), one obtains

$$
\begin{aligned}
& \left\langle\mathbf{n}\left|A_{L}^{\dagger}\left(\omega_{\ell}\right) \sigma_{z}^{(k)} A_{L}\left(\omega_{\ell}\right)\right| \mathbf{n}\right\rangle=u_{1 \ell}^{2} \delta_{1 n_{\ell}}\left\langle\mathbf{n}_{0_{\ell}}\left|\sigma_{z}^{(k)}\right| \mathbf{n}_{0_{\ell}}\right\rangle, \\
& \left\langle\mathbf{n}\left|\left\{A_{L}^{\dagger}\left(\omega_{\ell}\right) A_{L}\left(\omega_{\ell}\right), \sigma_{z}^{(k)}\right\}\right| \mathbf{n}\right\rangle=u_{1 \ell}^{2} \delta_{1 n_{\ell}}\left\langle\mathbf{n}\left|\sigma_{z}^{(k)}\right| \mathbf{n}\right\rangle, \\
& \left\langle\mathbf{n}\left|A_{L}\left(\omega_{\ell}\right) \sigma_{z}^{(k)} A_{L}^{\dagger}\left(\omega_{\ell}\right)\right| \mathbf{n}\right\rangle=u_{1 \ell}^{2} \delta_{0 n_{\ell}}\left\langle\mathbf{n}_{1_{\ell}}\left|\sigma_{z}^{(k)}\right| \mathbf{n}_{1_{\ell}}\right\rangle, \\
& \left\langle\mathbf{n}\left|\left\{A_{L}\left(\omega_{\ell}\right) A_{L}^{\dagger}\left(\omega_{\ell}\right), \sigma_{z}^{(k)}\right\}\right| \mathbf{n}\right\rangle=u_{1 \ell}^{2} \delta_{0 n_{\ell}}\left\langle\mathbf{n}\left|\sigma_{z}^{(k)}\right| \mathbf{n}\right\rangle .
\end{aligned}
$$

Then, from Eqs. (26) and (36), it follows that

$$
\sigma_{z}^{(k)}=-1+2 \sum_{r, s=1}^{N} u_{k r} u_{k s} b_{r}^{\dagger} b_{s},
$$

and hence, using Eqs. (32)-(34),

$$
\begin{aligned}
& \left\langle\mathbf{n}\left|\sigma_{z}^{(k)}\right| \mathbf{n}\right\rangle=2 \sum_{r=1}^{N} n_{r} u_{k r}^{2}-1, \\
& \left\langle\mathbf{n}_{0_{\ell}}\left|\sigma_{z}^{(k)}\right| \mathbf{n}_{0_{\ell}}\right\rangle-\left\langle\mathbf{n}\left|\sigma_{z}^{(k)}\right| \mathbf{n}\right\rangle=-2 n_{\ell} u_{k \ell}^{2}, \\
& \left\langle\mathbf{n}_{1_{\ell}}\left|\sigma_{z}^{(k)}\right| \mathbf{n}_{1_{\ell}}\right\rangle-\left\langle\mathbf{n}\left|\sigma_{z}^{(k)}\right| \mathbf{n}\right\rangle=2\left(1-n_{\ell}\right) u_{k \ell}^{2} .
\end{aligned}
$$

Inserting the last three expressions into Eqs. (E2)-(E7) yields

$$
\left\langle\mathbf{n}\left|\widetilde{\mathbb{D}}_{\omega_{\ell}}^{(L)}\left[\sigma_{z}^{(k)}\right]\right| \mathbf{n}\right\rangle=u_{k \ell}^{2} u_{1 \ell}^{2}\left(\widetilde{C}_{\omega_{\ell}}^{(L)} \delta_{0 n_{\ell}}-C_{\omega_{\ell}}^{(L)} \delta_{1 n_{\ell}}\right),
$$

and hence, finally, using the stationary-state eigenvalues in Eqs. (54)-(57) and the explicit form of the constants $C_{\omega_{\ell}}^{(L)}$ and $\widetilde{C}_{\omega_{\ell}}^{(L)}$ in Eqs. (15) and (16), the sink and/or source contribution $\mathfrak{Q}_{L}^{(k)}$ in Eq. (73) ensues. Similar arguments lead to $\mathfrak{Q}_{R}^{(k)}$ in Eq. (74).

\section{APPENDIX F: STATIONARY-STATE EIGENVALUES FOR TWO- AND THREE-SPIN CHAINS \\ 1. Two-spin chain}

For two spins and $N=2$, the four two-digit strings are, in antilexicographic order, (00), (10), (01), and (11). Moreover, using Eq. (82) their combinadic ordering can be shown to be the same; namely,

$$
(00) \leftrightarrow \mathcal{N}_{0}=1, \quad\left\{\begin{array}{l}
(10) \leftrightarrow \mathcal{N}_{1}=1 \\
(01) \leftrightarrow \mathcal{N}_{1}=2, \quad(11) \leftrightarrow \mathcal{N}_{2}=1 .
\end{array}\right.
$$

Then, the combinadic list of the eigenvalues $\Lambda_{n_{1} n_{2}}$ of the stationary state $\rho_{\infty}$ in Eq. (83) is as follows:

$$
\begin{aligned}
& \mathcal{L}_{1}^{(0)}= \Lambda_{00}=\frac{R_{0}^{(1)} R_{0}^{(2)}}{R_{1} R_{2}}, \quad \text { for } p=0, \\
& \mathcal{L}_{1}^{(1)}=\Lambda_{10}=\frac{R_{1}^{(1)} R_{0}^{(2)}}{R_{1} R_{2}}, \quad \mathcal{L}_{2}^{(1)}=\Lambda_{01}=\frac{R_{0}^{(1)} R_{1}^{(2)}}{R_{1} R_{2}}, \\
& \quad \text { for } p=1,
\end{aligned}
$$

and

$$
\mathcal{L}_{1}^{(2)}=\Lambda_{11}=\frac{R_{1}^{(1)} R_{1}^{(2)}}{R_{1} R_{2}}, \quad \text { for } p=2 .
$$

\section{Three-spin chains}

For $N=3$, let us consider the antilexicographic ordering of the eight three-digit strings:

$$
\text { (000), (100), (010), (110), (001), (101), (011), (111). }
$$

Application of Eq. (82) shows that this ordering and the combinadic ordering coincide, in the sense that

$$
\begin{aligned}
& (000) \leftrightarrow \mathcal{N}_{0}=1, \quad\left\{\begin{array}{l}
(100) \leftrightarrow \mathcal{N}_{1}=1, \\
(010) \leftrightarrow \mathcal{N}_{1}=2, \\
(001) \leftrightarrow \mathcal{N}_{1}=3 ;
\end{array}\right. \\
& \left\{\begin{array}{l}
(110) \leftrightarrow \mathcal{N}_{2}=1, \\
(101) \leftrightarrow \mathcal{N}_{2}=2, \\
(011) \leftrightarrow \mathcal{N}_{2}=3,
\end{array}\right.
\end{aligned}
$$

Then, for a three-spin chain, the eigenvalues $\Lambda_{n_{1} n_{2} n_{3}}$ of the stationary state $\rho_{\infty}$ in Eq. (83) are

$$
\mathcal{L}_{1}^{(0)}=\Lambda_{000}=\frac{R_{0}^{(1)} R_{0}^{(2)} R_{0}^{(3)}}{R^{(1)} R^{(2)} R^{(3)}}
$$


for $p=0$, while for $p=1$,

$$
\begin{gathered}
\mathcal{L}_{1}^{(1)}=\Lambda_{100}=\frac{R_{1}^{(1)} R_{0}^{(2)} R_{0}^{(3)}}{R_{1} R_{2} R_{3}}, \\
\mathcal{L}_{2}^{(1)}=\Lambda_{010}=\frac{R_{0}^{(1)} R_{1}^{(2)} R_{0}^{(3)}}{R_{1} R_{2} R_{3}}, \\
\mathcal{L}_{3}^{(1)}=\Lambda_{001}=\frac{R_{0}^{(1)} R_{0}^{(2)} R_{1}^{(3)}}{R_{1} R_{2} R_{3}},
\end{gathered}
$$

for $p=2$,

$$
\begin{gathered}
\mathcal{L}_{1}^{(2)}=\Lambda_{110}=\frac{R_{1}^{(1)} R_{1}^{(2)} R_{0}^{(3)}}{R_{1} R_{2} R_{3}}, \\
\mathcal{L}_{2}^{(2)}=\Lambda_{101}=\frac{R_{1}^{(1)} R_{0}^{(2)} R_{1}^{(3)}}{R_{1} R_{2} R_{3}}, \\
\mathcal{L}_{3}^{(2)}=\Lambda_{011}=\frac{R_{0}^{(1)} R_{1}^{(2)} R_{1}^{(3)}}{R_{1} R_{2} R_{3}}
\end{gathered}
$$

and, finally, for $p=3$,

$$
\mathcal{L}_{1}^{(3)}=\Lambda_{111}=\frac{R_{1}^{(1)} R_{1}^{(2)} R_{1}^{(3)}}{R_{1} R_{2} R_{3}}
$$

The quantities

$$
R_{0}^{(\ell)}=\left[h_{L}\left(\omega_{\ell}\right)\right]^{2}\left(1+n_{L}\left(\omega_{\ell}\right)\right)+\left[h_{R}\left(\omega_{\ell}\right)\right]^{2}\left(1+n_{R}\left(\omega_{\ell}\right)\right)
$$

and

and

$$
R_{1}^{(\ell)}=\left[h_{L}\left(\omega_{\ell}\right)\right]^{2} n_{L}\left(\omega_{\ell}\right)+\left[h_{R}\left(\omega_{\ell}\right)\right]^{2} n_{R}\left(\omega_{\ell}\right)
$$

in Eq. (56) coincide with the quantities $s_{\ell}$ and $\tau_{\ell}$, respectively, in Ref. [50]. According to the correspondence of the frequencies $\omega_{1,2,3}$ in Eq. (C6) with the frequencies $\omega_{1,0,2}$ in Ref. [50], the following correspondence arises among the stationary-state eigenvalues $\Lambda_{\mathbf{n}}$ and the eigenvalues $\mu_{k}$ in Ref. [50]:

$$
\begin{aligned}
& \mathcal{L}_{1}^{(0)}=\mu_{2}, \quad \mathcal{L}_{1}^{(1)}=\mu_{7}, \quad \mathcal{L}_{2}^{(1)}=\mu_{4}, \quad \mathcal{L}_{3}^{(1)}=\mu_{6}, \\
& \mathcal{L}_{1}^{(2)}=\mu_{5}, \quad \mathcal{L}_{2}^{(2)}=\mu_{3}, \quad \mathcal{L}_{3}^{(2)}=\mu_{8}, \quad \mathcal{L}_{1}^{(3)}=\mu_{1} .
\end{aligned}
$$

\section{APPENDIX G: STATIONARY STATE BY COMBINADIC ORDERING}

According to the combinadic ordering introduced in Sec. VI A, the stationary state $\rho_{\infty}$ may be written as

$$
\rho_{\infty}=\sum_{p=0}^{N} \sum_{\mathcal{N}_{p}=1}^{\left(\begin{array}{l}
N \\
p
\end{array}\right)} \mathcal{L}_{\mathcal{N}_{p}}^{(p)}\left|\mathcal{N}_{p}\right\rangle\left\langle\mathcal{N}_{p}\right|
$$

where $\mathcal{L}_{\mathcal{N}_{p}}^{(p)}$ denotes the eigenvalue $\Lambda_{\mathbf{n}}$ in Eq. (54) corresponding to the binary $N$-tuple $\mathbf{n}$ with $p 1$ 's, indexed by the combinadic integer $\mathcal{N}_{p}$. Note that, for any fixed $p=0,1, \ldots, N$, the integers $\mathcal{N}_{p}$ in Eq. (82) correspond to the fermionic excitations of the modes $i_{1}<\cdots<i_{p}$ of type $b$. Indeed, $\mathcal{N}_{p}$ identifies the binary $N$-tuple $\mathbf{n}$, where $n_{i_{1}}=\cdots=n_{i_{p}}=1$, while the remaining $n_{j}$ vanish. We can thus consistently set

$$
\left|\mathcal{N}_{p}\right\rangle=b_{i_{1}}^{\dagger} \cdots b_{i_{p}}^{\dagger}|v\rangle
$$

Then, using Eq. (29), one writes

$$
\left|\mathcal{N}_{p}\right\rangle=\sum_{j_{1}, j_{2}, \ldots, j_{p}} u_{i_{1} j_{1}} u_{i_{2} j_{2}} \cdots u_{i_{p} j_{p}} a_{j_{1}}^{\dagger} a_{j_{2}}^{\dagger} \cdots a_{j_{p}}^{\dagger}|v\rangle
$$

However, unlike the indices $i_{1}, \ldots, i_{p}$, the indices $j_{1}, j_{2}, \ldots, j_{p}$ are in general not ordered; reordering them such that $j_{1}<j_{2}<\cdots<j_{p}$ yields

$$
\left|\mathcal{N}_{p}\right\rangle=\sum_{j_{1}<j_{2}<\ldots<j_{p}} D_{j_{1}<\cdots<j_{p}}^{i_{1}<\cdots<i_{p}} a_{j_{1}}^{\dagger} a_{j_{2}}^{\dagger} \cdots a_{j_{p}}^{\dagger}|v\rangle
$$

where

$$
D_{j_{1}<\cdots<j_{p}}^{i_{1}<\cdots<i_{p}}=\operatorname{det}\left(U_{j_{1}<\cdots<j_{p}}^{i_{1}<\cdots<i_{p}}\right)
$$

is the determinant of the $p \times p$ submatrix $U_{j_{1}<\cdots<j_{p}}^{i_{1}<\cdots<i_{p}}$ of the orthogonal and symmetric matrix $U$ in Remark 3, with $p$ rows indexed by $i_{1}<\cdots<i_{p}$ and $p$ columns by $j_{1}<$ $\cdots<j_{p}$. Its entries are thus given by

$$
\left(U_{j_{1}<\cdots<j_{p}}^{i_{1}<\cdots<i_{p}}\right)_{a b}=u_{i_{a j} j_{b}}=\sqrt{\frac{2}{N+1}} \sin \left(\frac{\pi i_{a j} j_{b}}{N+1}\right) .
$$

For the same spin chain but with periodic boundary conditions, similar expressions are derived in Ref. [52].

Remark 5: For $p=0$, all $n_{j}=0$ and hence there are no $i_{\ell}$ and $j_{\ell}$ to choose and one sets $U_{j_{1}<\cdots<j_{p}}^{i_{1}<\cdots<i_{p}}=1$. Instead, if only $n_{i_{\ell}}=1$, then the matrices $U_{j_{1}<\cdots<j_{p}}^{i_{1}<\cdots<i_{p}}$ reduce to the scalars $U_{j_{\ell}}^{i_{\ell}}=u_{i_{\ell} j_{\ell}}$. Finally, if all $n_{j}=1$, then there is only 
one contributing matrix, $U_{12 \cdots N}^{12 \cdots N}=U$, and $\operatorname{Det}(U)=-1$. Unlike the matrix $U$, the submatrices $U_{j_{1}<\cdots<j_{p}}^{i_{1}<\cdots<i_{p}}$ are not symmetric; however,

$$
D_{j_{1}<\cdots<j_{p}}^{i_{1}<\cdots<i_{p}}=D_{i_{1}<\cdots<i_{p}}^{j_{1}<\cdots<j_{p}}
$$

It is convenient to introduce the $\left(\begin{array}{l}N \\ p\end{array}\right) \times\left(\begin{array}{l}N \\ p\end{array}\right)$ matrices $\mathcal{D}^{(p)}$, where $\mathcal{D}^{(0)}=1$ and $\mathcal{D}^{(N)}=-1$ are scalars; otherwise, $\mathcal{D}^{(p)}$ has entries $\mathcal{D}_{\mathcal{N}_{p}^{\prime} \mathcal{N}_{p}^{\prime \prime}}^{(p)}$ corresponding to the determinants $D_{i_{1}^{\prime \prime}<\cdots<i_{p}^{\prime \prime}}^{i_{1}^{\prime}<\cdots<i_{p}^{\prime}}$, where $\mathcal{N}_{p}^{\prime}$ identifies an $N$-tuple with 1 's at sites $i_{1}^{\prime}<\cdots<i_{p}^{\prime}$ and $\mathcal{N}_{p}^{\prime \prime}$ identifies an $N$-tuple with 1 's at sites $i_{1}^{\prime \prime}<\cdots<i_{p}^{\prime \prime}$. Because of Eq. (G7), the matrices $\mathcal{D}^{(p)}$ are symmetric for all $p=0,1, \ldots, N$.

Since in the spin representation $|v\rangle=|\downarrow\rangle^{\otimes N}$, as shown in Appendix $\mathrm{H}$, setting $|\uparrow\rangle=|1\rangle_{S}$ and $|\downarrow\rangle=|0\rangle_{S}$ so that $\sigma_{+}|0\rangle_{S}=|1\rangle_{S}$, one can express the fermionic states $\left|\mathcal{N}_{p}\right\rangle$ with $p$ excitations at sites $i_{1}<\cdots<i_{p}$ as linear combinations of the spin states $\left|\mathcal{N}_{p}^{\prime}\right\rangle_{S}$ with $p$ spins flipped up at the sites $i_{1}^{\prime}<\cdots<i_{p}^{\prime}$ identified by the combinadic index $\mathcal{N}_{p}^{\prime}$. Then, with respect to the standard spin basis, the stationary state $\rho_{\infty}$ in Eq. (G1) reads

$$
\rho_{\infty}=\sum_{p=0}^{N} \sum_{\mathcal{N}_{p}^{\prime}, \mathcal{N}_{p}^{\prime \prime}} \mathcal{S}_{\mathcal{N}_{p}^{\prime} \mathcal{N}_{p}^{\prime \prime}}^{(p)}\left|\mathcal{N}_{p}^{\prime}\right\rangle_{S S}\left\langle\mathcal{N}_{p}^{\prime \prime}\right|
$$

where

$$
\mathcal{S}_{\mathcal{N}_{p}^{\prime} \mathcal{N}_{p}^{\prime \prime}}^{(p)}:=\sum_{\mathcal{N}_{p}} \mathcal{L}_{\mathcal{N}_{p}} \mathcal{D}_{\mathcal{N}_{p} \mathcal{N}_{p}^{\prime}}^{(p)} \mathcal{D}_{\mathcal{N}_{p} \mathcal{N}_{p}^{\prime \prime}}^{(p)}
$$

From the two previous expressions, it follows that the stationary state can be identified by the block-diagonal matrix

$$
\mathcal{S}=\bigoplus_{p=0}^{N} \mathcal{S}^{(p)}, \quad \mathcal{S}^{(p)}=\mathcal{D}^{(p)} \mathcal{L}^{(p)} \mathcal{D}^{(p)}, \quad \mathcal{D}=\bigoplus_{p=0}^{N} \mathcal{D}^{(p)}
$$

where $\mathcal{L}^{(p)}$ is the diagonal matrix the entries of which are the eigenvalues in Eq. (54), labeled by the combinadic integers $\mathcal{N}_{p}$, while the matrices $\mathcal{D}^{(p)}$ are as in the previous remark.

\section{APPENDIX H: STATIONARY STATE IN THE SPIN REPRESENTATION}

Given the combinadic labeling given in Eq. (G2) of the energy eigenstates in the fermionic representation, from
Eq. (23) one obtains

$$
\begin{aligned}
\left|\mathcal{N}_{p}\right\rangle= & \sum_{j_{1}<j_{2}<\ldots<j_{p}} D_{j_{1}<\cdots<j_{p}}^{i_{1}<\cdots<i_{p}}\left(\prod_{k=1}^{j_{1}-1}\left(-\sigma_{z}^{(k)}\right)^{p}\right) \\
& \left.\left.\times\left(-\sigma_{+}^{\left(j_{1}\right)} \sigma_{z}^{j_{1}}\right)\right)\left(\prod_{k=1}^{j_{2}-1}\left(-\sigma_{z}^{(k)}\right)^{p-1}\right)\left(-\sigma_{+}^{\left(j_{2}\right)} \sigma_{z}^{j_{2}}\right)\right) \\
& \times \cdots\left(-\sigma_{+}^{\left(j_{p-1}\right.} \sigma_{z}^{\left(j_{p-1}\right)}\right)\left(\prod_{k=1}^{j_{1}-1}\left(-\sigma_{z}^{(k)}\right)\right) \sigma_{+}^{\left(j_{p}\right)}|v\rangle .
\end{aligned}
$$

Since $\sigma_{+} \sigma_{z}=-\sigma_{+}$, one rewrites

$$
\left|\mathcal{N}_{p}\right\rangle=\sum_{j_{1}<\ldots<j_{p}} D_{j_{1}<\cdots<j_{p}}^{i_{1}<\cdots<i_{p}} S_{j_{1} \cdots j_{p}}|v\rangle
$$

where

$$
\begin{aligned}
S_{j_{1} \cdots j_{p}}|v\rangle & \left(\prod_{k=1}^{j_{1}-1}\left(-\sigma_{z}^{(k)}\right)^{p}\right) \sigma_{+}^{\left(j_{1}\right)}\left(\prod_{k=j_{1}+1}^{j_{2}-1}\left(-\sigma_{z}^{(k)}\right)^{p-1}\right) \sigma_{+}^{\left(j_{2}\right)} \\
& \times \cdots \sigma_{+}^{\left(j_{p-1}\right)}\left(\prod_{k=j_{p-1}+1}^{j_{p}-1}\left(-\sigma_{z}^{(k)}\right)\right) \sigma_{+}^{\left(j_{p}\right)}|v\rangle
\end{aligned}
$$

Because of Eq. (25), with $|\uparrow\rangle=|1\rangle_{S}$ and $|\downarrow\rangle=|0\rangle_{S}$, one writes

$$
S_{j_{1} \cdots j_{p}}|v\rangle=\left|\mathcal{N}_{p}^{\prime}\right\rangle_{S}
$$

where, according to Eq. (82), $\mathcal{N}_{p}^{\prime}$ uniquely identifies the $N$ tuple with $n_{j_{1}}^{\prime}=\cdots=n_{j_{p}}^{\prime}=1$ and $n_{k}^{\prime}=0$ otherwise: such an $N$-tuple corresponds in its turn to a spin state vector with spin up at the sites $j_{1}<\cdots<j_{p}$ and down at all other sites. Thus, with respect to the standard spin basis, the stationary states can be recast as in Eqs. (G8) and (G9).

In order to rewrite $\rho_{\infty}$ as a tensor product of on-site spin operators, one starts from the projectors

$$
\begin{aligned}
\left|\mathcal{N}_{p}\right\rangle\left\langle\mathcal{N}_{p}\right|= & \sum_{j_{1}<\ldots<j_{p}} \sum_{k_{1}<\cdots<k_{p}} D_{j_{1}<\cdots<j_{p}}^{i_{1}<\cdots<i_{p}} D_{k_{1}<\cdots<k_{p}}^{i_{1}<\cdots<i_{p}} \\
& \times S_{j_{1} \cdots j_{p}}|v\rangle\langle v| S_{k_{1} \cdots k_{p}}^{\dagger}
\end{aligned}
$$

Then, writing $|v\rangle\langle v|=\prod_{\ell=1}^{N}\left(1-\sigma_{z}^{(\ell)}\right) / 2$ and using the fact that

$$
\sigma_{+} \frac{1-\sigma_{z}}{2}=\sigma_{+}, \quad\left(-\sigma_{z}\right)^{k} \frac{1-\sigma_{z}}{2}=\frac{1-\sigma_{z}}{2} \quad \forall k,
$$


one recasts $S_{j_{1} \cdots j_{p}}|v\rangle\langle v|$ as

$$
\begin{aligned}
& \left(\prod_{k=1}^{j_{1}-1} \frac{1-\sigma_{z}^{(k)}}{2}\right) \sigma_{+}^{\left(j_{1}\right)}\left(\prod_{k=j_{1}+1}^{j_{2}-1} \frac{1-\sigma_{z}^{(k)}}{2}\right) \sigma_{+}^{\left(j_{2}\right)} \cdots \sigma_{+}^{\left(j_{p-1}\right)} \\
& \quad \times\left(\prod_{k=j_{p-1}+1}^{j_{p}-1} \frac{1-\sigma_{z}^{(k)}}{2}\right) \sigma_{+}^{\left(j_{p}\right)} .
\end{aligned}
$$

As done before, using Eq. (82), we identify any given set of indices $j_{1}<\cdots<j_{p}$ and the corresponding $N$-tuple with $n_{j_{1}}=\cdots=n_{j_{p}}$ by the unique combinadic integer $\mathcal{N}_{p}^{\prime}$ and hence

$$
\begin{aligned}
& S_{j_{1} \cdots j_{p}}|v\rangle\langle v|=\mathcal{Z}_{\mathcal{N}_{p}^{\prime}}^{(p)} \\
& \left|\mathcal{N}_{p}\right\rangle\left\langle\mathcal{N}_{p}\right|=\sum_{\mathcal{N}_{p}^{\prime}, \mathcal{N}_{p}^{\prime \prime}} \mathcal{D}_{\mathcal{N}_{p} \mathcal{N}_{p}^{\prime}}^{(p)} \mathcal{D}_{\mathcal{N}_{p} \mathcal{N}_{p}^{\prime \prime}}^{(p)} \mathcal{Z}_{\mathcal{N}_{p}^{\prime}}^{(p)}\left(\mathcal{Z}_{\mathcal{N}_{p}^{\prime \prime}}^{(p)}\right)^{\dagger},
\end{aligned}
$$

where, setting $X_{0}^{(\ell)}=\left(1-\sigma_{z}^{(\ell)}\right) / 2$ and $X_{1}^{(\ell)}=\sigma_{+}^{(\ell)}$,

$$
\mathcal{Z}_{\mathcal{N}_{p}^{\prime}}^{(p)}\left(\mathcal{Z}_{\mathcal{N}_{p}^{\prime \prime}}^{(p)}\right)^{\dagger}=\prod_{\ell=1}^{N}\left(X_{n_{\ell}^{\prime}}^{(\ell)}\left(X_{n_{\ell}^{\prime \prime}}^{(\ell)}\right)^{\dagger}\right)
$$

It thus follows that in spin-operatorial form, the stationary state reads as in Eq. (84).

\section{APPENDIX I: SPIN REPRESENTATION OF THE STATIONARY STATE FOR TWO- AND THREE-SPIN CHAINS}

\section{Two-spin chain}

The case of a two-spin chain is the simplest: as there can be at most two spins up, the values of $p$ are 0,1 , and 2 . Then, $\mathcal{D}^{(0)}=1, \mathcal{D}^{(2)}=-1$ and $\mathcal{D}^{(1)}=U$ and hence, using Eqs. (F2)-(F4), from Eq. (G10) one obtains $\mathcal{S}^{(0)}=\Lambda_{00}$, $\mathcal{S}^{(2)}=\Lambda_{11}$ and

$$
\begin{aligned}
\mathcal{S}^{(1)} & =U\left(\begin{array}{cc}
\Lambda_{10} & 0 \\
0 & \Lambda_{01}
\end{array}\right) U \\
& =\frac{1}{2}\left(\begin{array}{cc}
\Lambda_{10}+\Lambda_{01} & \Lambda_{10}-\Lambda_{01} \\
\Lambda_{10}-\Lambda_{01} & \Lambda_{10}+\Lambda_{01}
\end{array}\right) .
\end{aligned}
$$

Finally, using Eqs. (F2)-(F4), Eq. (84) yields

$$
\begin{aligned}
\rho_{\infty}= & \Lambda_{00} P_{-} \otimes P_{+}+\Lambda_{11} P_{+} \otimes P_{+} \\
& +\frac{\Lambda_{10}+\Lambda_{01}}{2}\left(P_{+} \otimes P_{-}+P_{-} \otimes P_{+}\right) \\
& +\frac{\Lambda_{10}-\Lambda_{01}}{2}\left(\sigma_{+} \otimes \sigma_{+}+\sigma_{-} \otimes \sigma_{+}\right) .
\end{aligned}
$$

The stationary state in the standard spin basis $|\uparrow \uparrow\rangle,|\uparrow \downarrow\rangle$, $|\downarrow \uparrow\rangle$, and $|\downarrow \downarrow\rangle$ explicitly reads

$$
\rho_{\infty}=\frac{1}{2}\left(\begin{array}{cccc}
2 \Lambda_{11} & 0 & 0 & 0 \\
0 & \Lambda_{10}+\Lambda_{01} & \Lambda_{10}-\Lambda_{01} & 0 \\
0 & \Lambda_{10}-\Lambda_{01} & \Lambda_{10}+\Lambda_{01} & 0 \\
0 & 0 & 0 & 2 \Lambda_{00}
\end{array}\right)
$$

and is thus a so-called $X$ state. Its entanglement content is measured by the concurrence $[62,63]$, which has, in this special case, the analytic expression:

$$
C\left(\rho_{\infty}\right)=\max \left(0,\left|\Lambda_{10}-\Lambda_{01}\right|-2 \sqrt{\Lambda_{00} \Lambda_{11}}\right) .
$$

In the case where $h_{L, R}\left(\omega_{1,2}\right)=h$, from Eq. (58) one obtains

$$
\begin{aligned}
& \Lambda_{00}=\frac{1}{4} \frac{2+N_{L R}\left(\omega_{1}\right)}{1+N_{L R}\left(\omega_{1}\right)} \frac{2+N_{L R}\left(\omega_{2}\right)}{1+N_{L R}\left(\omega_{2}\right)}, \\
& \Lambda_{10}=\frac{1}{4} \frac{N_{L R}\left(\omega_{1}\right)}{1+N_{L R}\left(\omega_{1}\right)} \frac{2+N_{L R}\left(\omega_{2}\right)}{1+N_{L R}\left(\omega_{2}\right)}, \\
& \Lambda_{01}=\frac{1}{4} \frac{2+N_{L R}\left(\omega_{1}\right)}{1+N_{L R}\left(\omega_{1}\right)} \frac{N_{L R}\left(\omega_{2}\right)}{1+N_{L R}\left(\omega_{2}\right)}, \\
& \Lambda_{11}=\frac{1}{4} \frac{N_{L R}\left(\omega_{1}\right)}{1+N_{L R}\left(\omega_{1}\right)} \frac{N_{L R}\left(\omega_{2}\right)}{1+N_{L R}\left(\omega_{2}\right)},
\end{aligned}
$$

where we set $N_{L R}(\omega):=n_{L}(\omega)+n_{R}(\omega)$. Then, one finds entanglement in the stationary state whenever $\left|N_{L R}\left(\omega_{1}\right)-N_{L R}\left(\omega_{2}\right)\right|$ is larger than

$$
2 \sqrt{\left(2+N_{L R}\left(\omega_{1}\right)\right)\left(2+N_{L R}\left(\omega_{2}\right)\right) N_{L R}\left(\omega_{1}\right) N_{L R}\left(\omega_{2}\right)} .
$$

\section{Three-spin chain}

For $N=3$, the number of 1's in the binary digits of length 3 is $p=0,1,2$, and 3 , the integers $1 \leq i_{1}<$ $\cdots<i_{p} \leq N$ denoting the sites at which the 1's occur. If there are no 1 's, such as when $p=0$, we set $i_{0}=0$ : the following 1's are then the possible strings:

$(000): i_{0}=0, \quad \begin{cases}(100): & i_{1}=1, \\ (010): & i_{1}=2, \\ (001): & i_{1}=3,\end{cases}$

$\begin{cases}(110): & i_{1}=1, i_{2}=2, \\ (101): & i_{1}=1, i_{2}=3, \quad(111): i_{1}=1, i_{2}=2, i_{3}=3 . \\ (011): & i_{1}=2, i_{2}=3,\end{cases}$

The binary strings above are listed antilexicographically: it turns out that their combinadic indices according to 
Eq. (82) provide the same ordering:

$$
\begin{aligned}
& (000) \leftrightarrow \mathcal{N}_{0}=1, \\
& (100) \leftrightarrow \mathcal{N}_{1}=1, \quad(010) \leftrightarrow \mathcal{N}_{1}=2, \quad(001) \leftrightarrow \mathcal{N}_{1}=3, \\
& (110) \leftrightarrow \mathcal{N}_{2}=1, \quad(101) \leftrightarrow \mathcal{N}_{2}=2, \quad(011) \leftrightarrow \mathcal{N}_{2}=3, \\
& (111) \leftrightarrow \mathcal{N}_{3}=1 .
\end{aligned}
$$

The $p \times p$ matrices $\mathcal{D}^{(p)}$ appearing in Eq. (G10) have entries $\mathcal{D}_{\mathcal{N}_{p}^{\prime} \mathcal{N}_{p}^{\prime \prime}}^{(p)}$ that are the determinants of the submatrices $U_{j_{1}<\cdots j_{p}}^{i_{1}<\cdots p_{p}}$ of rank $p$ that are obtained from the unitary matrix

$$
U=\left[u_{i j}\right]=\frac{1}{2}\left(\begin{array}{ccc}
1 & \sqrt{2} & 1 \\
\sqrt{2} & 0 & -\sqrt{2} \\
1 & -\sqrt{2} & 1
\end{array}\right)
$$

by choosing the rows indexed by $i_{1}<\cdots<i_{p}$ and the columns indexed by $j_{1}<\cdots j_{p}$. Some instances of the various entries follow:

$$
\begin{aligned}
& \mathcal{D}_{1}^{(0)}=\operatorname{det}\left(U_{0}^{0}\right)=1, \\
& \mathcal{D}_{1}^{(3)}=\operatorname{det}\left(U_{123}^{123}\right)=-1, \\
& \mathcal{D}_{11}^{(1)}=\operatorname{det}\left(U_{1}^{1}\right)=u_{11}=\frac{1}{2}, \\
& \mathcal{D}_{11}^{(2)}=\operatorname{det}\left(U_{12}^{12}\right)=\left|\begin{array}{ll}
u_{11} & u_{12} \\
u_{21} & u_{22}
\end{array}\right|=-\frac{1}{2} .
\end{aligned}
$$

It follows that $\mathcal{D}^{(1)}=U$ and $\mathcal{D}^{(2)}=-U$.

Given the diagonal symmetric matrix in Eq. (83), consisting of the eigenvalues in Eq. (54) of the stationary state $\rho_{\infty}$, the entries of the blocks $\mathcal{S}^{(p)}$ of the matrix $\mathcal{S}$, which represents the three-spin stationary state with respect to the standard basis given in Eq. (G8), are (note that, due to Eq. (G7), $\mathcal{S}_{\mathcal{N}_{p}^{\prime} \mathcal{N}_{p}^{\prime \prime p}}^{(p)}=\mathcal{S}_{\mathcal{N}_{p}^{\prime \prime} \mathcal{N}_{p}^{\prime}}^{(p)}$ )

$$
\mathcal{S}_{11}^{(0)}=\mathcal{L}_{1}^{(0)}, \quad \mathcal{S}_{11}^{(3)}=\mathcal{L}_{1}^{(3)},
$$

for $p=0$ and $p=3$,

$$
\begin{aligned}
& \mathcal{S}_{11}^{(1)}=\mathcal{S}_{33}^{(1)}=\frac{\mathcal{L}_{1}^{(1)}+2 \mathcal{L}_{2}^{(1)}+\mathcal{L}_{3}^{(1)}}{4}, \\
& \mathcal{S}_{12}^{(1)}=\mathcal{S}_{23}^{(1)}=\frac{\mathcal{L}_{1}^{(1)}-\mathcal{L}_{3}^{(1)}}{2 \sqrt{2}}, \\
& \mathcal{S}_{13}^{(1)}=\frac{\mathcal{L}_{1}^{(1)}-2 \mathcal{L}_{2}^{(1)}+\mathcal{L}_{3}^{(1)}}{4}, \\
& \mathcal{S}_{22}^{(1)}=\frac{\mathcal{L}_{1}^{(1)}+\mathcal{L}_{3}^{(1)}}{2 \sqrt{2}},
\end{aligned}
$$

for $p=1$ and, for $p=2$,

$$
\begin{aligned}
& \mathcal{S}_{11}^{(2)}=\mathcal{S}_{33}^{(2)}=\frac{\mathcal{L}_{1}^{(2)}+2 \mathcal{L}_{2}^{(2)}+\mathcal{L}_{3}^{(2)}}{4}, \\
& \mathcal{S}_{12}^{(2)}=\mathcal{S}_{23}^{(2)}=\frac{\mathcal{L}_{1}^{(2)}-\mathcal{L}_{3}^{(2)}}{2 \sqrt{2}}, \\
& \mathcal{S}_{13}^{(2)}=\frac{\mathcal{L}_{1}^{(2)}-2 \mathcal{L}_{2}^{(2)}+\mathcal{L}_{3}^{(2)}}{4}, \\
& \mathcal{S}_{22}^{(2)}=\frac{\mathcal{L}_{1}^{(2)}+\mathcal{L}_{3}^{(2)}}{2} .
\end{aligned}
$$

Using Eq. (84) and the above expressions for $\mathcal{S}_{\mathcal{N}_{p}^{\prime} \mathcal{N}_{p}^{\prime \prime}}^{(p)}$, one recovers the following algebraic form for the diagonal contributions to the stationary state $\rho_{\infty}$ in the spin-operator representation:

$$
\begin{aligned}
\rho_{\infty}^{\text {diag }}= & \mathcal{L}_{1}^{(0)} P_{---}+\mathcal{L}_{1}^{(3)} P_{+++}+\frac{\mathcal{L}_{1}^{(1)}+\mathcal{L}_{3}^{(1)}}{2} P_{-+-} \\
& +\frac{\mathcal{L}_{1}^{(2)}+\mathcal{L}_{3}^{(2)}}{2} P_{+-+} \\
& +\frac{\mathcal{L}_{1}^{(1)}+2 \mathcal{L}_{2}^{(1)}+\mathcal{L}_{3}^{(1)}}{4}\left(P_{+--}+P_{--+}\right) \\
& +\frac{\mathcal{L}_{1}^{(2)}+2 \mathcal{L}_{2}^{(2)}+\mathcal{L}_{3}^{(2)}}{4}\left(P_{++-}+P_{-++}\right),
\end{aligned}
$$

where $\quad P_{i j k}=P_{i} \otimes P_{j} \otimes P_{k}, \quad i, j, k= \pm \quad$ and $\quad P_{ \pm}=$ $\left(1 \pm \sigma_{z}\right) / 2$. The off-diagonal contributions, on the other hand, read

$$
\begin{aligned}
\rho_{\infty}^{\text {off }}= & \frac{\mathcal{L}_{1}^{(1)}-\mathcal{L}_{3}^{(1)}}{2 \sqrt{2}}\left(\sigma_{+} \otimes \sigma_{-} \otimes P_{-}+P_{-} \otimes \sigma_{+} \otimes \sigma_{-}\right) \\
& +\frac{\mathcal{L}_{1}^{(2)}-\mathcal{L}_{3}^{(2)}}{2 \sqrt{2}}\left(P_{+} \otimes \sigma_{+} \otimes \sigma_{-}+\sigma_{+} \otimes \sigma_{-} \otimes P_{+}\right) \\
& +\frac{\mathcal{L}_{1}^{(1)}-2 \mathcal{L}_{2}^{(1)}+\mathcal{L}_{3}^{(1)}}{4} \sigma_{+} \otimes P_{-} \otimes \sigma_{-} \\
& +\frac{\mathcal{L}_{1}^{(2)}-2 \mathcal{L}_{2}^{(2)}+\mathcal{L}_{3}^{(2)}}{4} \sigma_{+} \otimes P_{+} \otimes \sigma_{-}+\text {h.c. }
\end{aligned}
$$

Due to the correspondence in Eqs. (F17) and (F18) among the eigenvalues of $\rho_{\infty}$ in Eqs. (F8)-(F14) with those in Ref. [50], it can be shown that the spin-operator expressions above coincide with those obtained there.

\section{APPENDIX J: TWO-SPIN ASYMPTOTIC DENSITY MATRICES}

The operation of tracing over the spins at sites different from $r$ and $s$ is denoted as $\operatorname{Tr}_{(r, s)}$. Considering the 
expression given in Eq. (84), one readily computes

$$
\begin{aligned}
\operatorname{Tr}_{(r, s)}\left(\prod_{\ell=1}^{N}\left(X_{n_{\ell}^{\prime}}^{(\ell)}\left(X_{n_{\ell}^{\prime \prime}}^{(\ell)}\right)^{\dagger}\right)\right) \\
=\left(\prod_{\ell \neq r, \ell \neq s} \operatorname{Tr}\left(X_{n_{\ell}^{\prime}}^{(\ell)}\left(X_{n_{\ell}^{\prime \prime}}^{(\ell)}\right)^{\dagger}\right)\right)\left(X_{n_{r}^{\prime}}^{(r)}\left(X_{n_{r}^{\prime \prime}}^{(r)}\right)^{\dagger}\right) \\
\times\left(X_{n_{s}^{\prime}}^{(s)}\left(X_{n_{s}^{\prime \prime}}^{(s)}\right)^{\dagger}\right) \\
=\left(\prod_{\ell \neq r, \ell \neq s} \delta_{n_{\ell}^{\prime} n_{\ell}^{\prime \prime}}\right)\left(X_{n_{r}^{\prime}} X_{n_{r}^{\prime \prime}}^{\dagger}\right) \otimes\left(X_{n_{s}^{\prime}} X_{n_{s}^{\prime \prime}}^{\dagger}\right),
\end{aligned}
$$

where, in the final two-spin expression, the reference to the spin sites has safely been neglected. We thus see that the partial trace reduces the double sum over all possible binary strings $\mathbf{n}^{\prime}$ and $\mathbf{n}^{\prime \prime}$ in Eq. (84) to a double sum over binary strings that have equal digits but, possibly, for the sites $r$ and $s$. We then denote by $\mathcal{N}_{p}^{(r s)}\left(n_{r}^{\prime}, n_{s}^{\prime}\right)$ and $\mathcal{N}_{p}^{(r s)}\left(n_{r}^{\prime \prime}, n_{s}^{\prime \prime}\right)$ the combinadic indices, given in Eq. (82), of the binary strings with $p 1$ 's that have the same entries $n_{j}$ everywhere except, possibly, for the sites $r$ and $s$.

With this notation, the two-spin density matrix formally reads

$$
\begin{aligned}
\rho_{(r, s)}:= & \operatorname{Tr}_{(r, s)}\left(\rho_{\infty}\right) \\
= & \sum_{n_{r}^{\prime}, n_{s}^{\prime} n_{r}^{\prime \prime}, n_{s}^{\prime \prime}} \sum_{p=0}^{N} \sum_{\mathcal{N}_{p}^{(r s)}\left(n_{r}^{\prime}, n_{s}^{\prime}\right) \mathcal{N}_{p}^{(r s)}\left(n_{r}^{\prime \prime}, n_{s}^{\prime \prime}\right)} \\
& \times \mathcal{S}_{\mathcal{N}_{p}^{(r)}}^{(p)}\left(n_{r}^{\prime}, n_{s}^{\prime}\right) \mathcal{N}_{p}^{(r s)}\left(n_{r}^{\prime \prime}, n_{s}^{\prime \prime}\right) \\
& \times\left(X_{n_{r}^{\prime}} X_{n_{r}^{\prime \prime}}^{\dagger}\right) \otimes\left(X_{n_{s}^{\prime}} X_{n_{s}^{\prime \prime}}^{\dagger}\right) .
\end{aligned}
$$

Since the $N$-tuples indexed by $\mathcal{N}_{p}^{(r s)}\left(n_{r}^{\prime \prime}, n_{s}^{\prime \prime}\right)$ have the same entries except, possibly, for the sites $r$ and $s$, it follows that the allowed values for $n_{r}^{\prime}, n_{s}^{\prime}$ and $n_{r}^{\prime \prime}, n_{s}^{\prime \prime}$ must satisfy $n_{r}^{\prime}+$ $n_{s}^{\prime}=n_{r}^{\prime \prime}+n_{s}^{\prime \prime}$. These latter 1 's and the corresponding twospin operators are as follows:

$$
\begin{aligned}
& \left\{\begin{array}{ll}
n_{r}^{\prime}=0, & n_{r}^{\prime \prime}=0 \\
n_{s}^{\prime}=0, & n_{s}^{\prime \prime}=0
\end{array}: \quad \frac{1-\sigma_{z}}{2} \otimes \frac{1-\sigma_{z}}{2},\right. \\
& \left\{\begin{array}{ll}
n_{r}^{\prime}=0, & n_{r}^{\prime \prime}=0 \\
n_{s}^{\prime}=1, & n_{s}^{\prime \prime}=1
\end{array}: \quad \frac{1-\sigma_{z}}{2} \otimes \frac{1+\sigma_{z}}{2},\right. \\
& \left\{\begin{array}{ll}
n_{r}^{\prime}=0, & n_{r}^{\prime \prime}=1 \\
n_{s}^{\prime}=1, & n_{s}^{\prime \prime}=0
\end{array}: \quad \sigma_{-} \otimes \sigma_{+},\right. \\
& \left\{\begin{array}{ll}
n_{r}^{\prime}=1, & n_{r}^{\prime \prime}=1 \\
n_{s}^{\prime}=0, & n_{s}^{\prime \prime}=0
\end{array}: \quad \frac{1+\sigma_{z}}{2} \otimes \frac{1-\sigma_{z}}{2}\right.
\end{aligned}
$$

and

$$
\begin{aligned}
& \left\{\begin{array}{ll}
n_{r}^{\prime}=1, & n_{r}^{\prime \prime}=0 \\
n_{s}^{\prime}=0, & n_{s}^{\prime \prime}=1
\end{array}: \quad \sigma_{+} \otimes \sigma_{-},\right. \\
& \left\{\begin{array}{ll}
n_{r}^{\prime}=1, & n_{r}^{\prime \prime}=1 \\
n_{s}^{\prime}=1, & n_{s}^{\prime \prime}=1
\end{array}: \quad \frac{1+\sigma_{z}}{2} \otimes \frac{1+\sigma_{z}}{2} .\right.
\end{aligned}
$$

Therefore, for all sites $1 \leq r<s \leq N$, one obtains

$$
\begin{aligned}
\rho_{(r, s)}= & a \frac{1+\sigma_{z}}{2} \otimes \frac{1+\sigma_{z}}{2}+b \frac{1+\sigma_{z}}{2} \otimes \frac{1-\sigma_{z}}{2} \\
& +c \sigma_{+} \otimes \sigma_{-}+c \sigma_{-} \otimes \sigma_{+} \\
& +d \frac{1-\sigma_{z}}{2} \otimes \frac{1+\sigma_{z}}{2}+e \frac{1-\sigma_{z}}{2} \otimes \frac{1-\sigma_{z}}{2},
\end{aligned}
$$

which, in the standard representation, corresponds to the $4 \times 4$ matrix in Eq. (86).

\section{APPENDIX K: TWO-SPIN CONCURRENCE}

\section{Two-spin concurrence in a three-spin chain}

Aided by the fact that, in the case $N=3$, the lexicographic and combinadic indices coincide as expressed by Eqs. (F5) and (F6), in order to find the indices $\mathcal{N}_{p}^{(r s)}\left(n^{\prime}, n^{\prime \prime}\right)$, one proceeds as follows. Since for $p=0$ the only possible string is (000),

$$
\begin{array}{ll}
\mathcal{N}_{0}^{(12)}(0,0)=1, & \mathcal{N}_{0}^{(12)}(0,1)=0 \\
\mathcal{N}_{0}^{(12)}(1,0)=0, & \mathcal{N}_{0}^{(12)}(1,1)=0 .
\end{array}
$$

For $p=1$, the possible strings are (100), (010), and (001); hence

$$
\begin{array}{ll}
\mathcal{N}_{1}^{(12)}(0,0)=3, & \mathcal{N}_{1}^{(12)}(0,1)=2, \\
\mathcal{N}_{1}^{(12)}(1,0)=1, & \mathcal{N}_{1}^{(12)}(1,1)=0 .
\end{array}
$$

For $p=2$, the possible strings are (110), (101), and (011); hence

$$
\begin{array}{ll}
\mathcal{N}_{2}^{(12)}(0,0)=0, & \mathcal{N}_{2}^{(12)}(0,1)=3 \\
\mathcal{N}_{2}^{(12)}(1,0)=2, & \mathcal{N}_{2}^{(12)}(1,1)=1 .
\end{array}
$$

Finally, for $p=3$, the only possible string is (111), so that

$$
\begin{aligned}
& \mathcal{N}_{3}^{(12)}(0,0)=0, \quad \mathcal{N}_{3}^{(12)}(0,1)=0, \\
& \mathcal{N}_{3}^{(12)}(1,0)=0, \quad \mathcal{N}_{3}^{(12)}(1,1)=1 .
\end{aligned}
$$


Similarly, for $r=1$ and $s=3$, that is, for computing the concurrence of the first and third spins, one finds that

$$
\begin{array}{ll}
\mathcal{N}_{0}^{(13)}(0,0)=1, & \mathcal{N}_{0}^{(13)}(0,1)=0, \\
\mathcal{N}_{0}^{(13)}(1,0)=0, & \mathcal{N}_{0}^{(13)}(1,1)=0, \\
\mathcal{N}_{1}^{(13)}(0,0)=2, & \mathcal{N}_{1}^{(13)}(0,1)=3, \\
\mathcal{N}_{1}^{(13)}(1,0)=1, & \mathcal{N}_{1}^{(13)}(1,1)=0, \\
\mathcal{N}_{2}^{(13)}(0,0)=0, & \mathcal{N}_{2}^{(13)}(0,1)=3, \\
\mathcal{N}_{2}^{(13)}(1,0)=1, & \mathcal{N}_{2}^{(13)}(1,1)=2, \\
\mathcal{N}_{3}^{(13)}(0,0)=0, & \mathcal{N}_{3}^{(13)}(0,1)=0, \\
\mathcal{N}_{3}^{(12)}(1,0)=0, & \mathcal{N}_{3}^{(12)}(1,1)=1 .
\end{array}
$$

Finally, for the concurrence of the second and third spins, setting $r=2$ and $s=3$, one finds that

$$
\begin{array}{ll}
\mathcal{N}_{0}^{(23)}(0,0)=1, & \mathcal{N}_{0}^{(23)}(0,1)=0, \\
\mathcal{N}_{0}^{(23)}(1,0)=0, & \mathcal{N}_{0}^{(23)}(1,1)=0, \\
\mathcal{N}_{1}^{(23)}(0,0)=1, & \mathcal{N}_{1}^{(23)}(0,1)=3, \\
\mathcal{N}_{1}^{(23)}(1,0)=2, & \mathcal{N}_{1}^{(23)}(1,1)=0, \\
\mathcal{N}_{2}^{(23)}(0,0)=0, & \mathcal{N}_{2}^{(23)}(0,1)=2, \\
\mathcal{N}_{2}^{(23)}(1,0)=1, & \mathcal{N}_{2}^{(23)}(1,1)=3, \\
\mathcal{N}_{3}^{(23)}(0,0)=0, & \mathcal{N}_{3}^{(23)}(0,1)=0, \\
\mathcal{N}_{3}^{(23)}(1,0)=0, & \mathcal{N}_{3}^{(23)}(1,1)=1 .
\end{array}
$$

Given the indices of the entries of the matrices $\mathcal{S}^{(p)}$ in Eq. (G10) that are necessary to compute the quantities $a$, $b$, and $c$ in Eqs. (88), (87), and (91), in the case of $r=1$, $s=2$ one finds that

$$
\begin{aligned}
& a=\mathcal{S}_{11}^{(2)}+\mathcal{S}_{11}^{(3)}, \\
& c=\mathcal{S}_{12}^{(1)}+\mathcal{S}_{23}^{(2)}, \\
& e=\mathcal{S}_{11}^{(0)}+\mathcal{S}_{33}^{(1)},
\end{aligned}
$$

while, in the case of $r=1$ and $s=3$,

$$
\begin{aligned}
& a=\mathcal{S}_{22}^{(2)}+\mathcal{S}_{11}^{(3)}, \\
& c=\mathcal{S}_{13}^{(1)}+\mathcal{S}_{13}^{(2)}, \\
& e=\mathcal{S}_{11}^{(0)}+\mathcal{S}_{22}^{(1)},
\end{aligned}
$$

and

$$
\begin{aligned}
& a=\mathcal{S}_{33}^{(2)}+\mathcal{S}_{11}^{(3)}, \\
& c=\mathcal{S}_{23}^{(1)}+\mathcal{S}_{12}^{(2)}, \\
& e=\mathcal{S}_{11}^{(0)}+\mathcal{S}_{11}^{(1)},
\end{aligned}
$$

in the case of $r=2$ and $s=3$. The insertion of Eqs. (I9)-(I17) into the previous expressions finally yields Eqs. (K34)-(K36) for both $r=1$ and $s=2$ and for $r=2$ and $s=3$, while Eqs. (K37)-(K39) result for $r=1$ and $s=3$.

In order to inspect the stationary entanglement of the first two spins, we set $r=1$ and $s=2$ and seek the combinadic indices $\mathcal{N}_{p}^{(12)}\left(n^{\prime}, n^{\prime \prime}\right)$ that select the entries of $\mathcal{S}^{(p)}$ to be used in Eqs. (87), (88), and (91). As shown above, the coefficients contributing to the concurrence $C_{1,2}$ in Eq. (92) are

$$
\begin{aligned}
& a=\frac{\mathcal{L}_{1}^{(2)}+2 \mathcal{L}_{2}^{(2)}+\mathcal{L}_{3}^{(2)}+4 \mathcal{L}_{1}^{(3)}}{4}, \\
& c=\frac{\mathcal{L}_{1}^{(1)}+\mathcal{L}_{1}^{(2)}-\mathcal{L}_{3}^{(1)}-\mathcal{L}_{3}^{(2)}}{2 \sqrt{2}}, \\
& e=\frac{\mathcal{L}_{1}^{(1)}+2 \mathcal{L}_{2}^{(1)}+\mathcal{L}_{3}^{(1)}+4 \mathcal{L}_{1}^{(0)}}{4} .
\end{aligned}
$$

In a similar fashion, again as shown in Appendix $J$, in the case of $C_{1,3}$, one finds that

$$
\begin{aligned}
& a=\frac{\mathcal{L}_{1}^{(2)}+2 \mathcal{L}_{1}^{(3)}+\mathcal{L}_{3}^{(2)}}{2}, \\
& c=\frac{\mathcal{L}_{1}^{(1)}-2 \mathcal{L}_{2}^{(1)}+\mathcal{L}_{3}^{(1)}+\mathcal{L}_{1}^{(2)}-2 \mathcal{L}_{2}^{(2)}+\mathcal{L}_{3}^{(2)}}{4}, \\
& e=\frac{\mathcal{L}_{1}^{(1)}+2 \mathcal{L}_{2}^{(0)}+\mathcal{L}_{3}^{(1)}}{4},
\end{aligned}
$$

while $C_{2,3}=C_{1,2}$, as the coefficients $a, c$, and $e$ coincide with those for $\rho_{(1,2)}$. The explicit expressions of the concurrences $C_{r, s}$ are not particularly suggestive and their dependence on $r$ and $s$ and on the bath temperatures must be addressed numerically: this is done in the next section for arbitrarily large chains. Here, we focus on the coefficient $c$, as its vanishing gives zero concurrence and thus excludes the existence of entanglement: in particular, we look at it under the simplifying assumption $h_{L, R}\left(\omega_{\ell}\right)=h$ for all $\ell=1,2,3$. Then, the eigenvalues $\mathcal{L}_{\mathcal{N}_{p}}^{(p)}$ in Eqs. (F7)-(F14) together with Eq. (58) yield

$$
c=\frac{N_{L R}\left(\omega_{1}\right)-N_{L R}\left(\omega_{3}\right)}{4 \sqrt{2}\left(1+N_{L R}\left(\omega_{1}\right)\right)\left(1+N_{L R}\left(\omega_{3}\right)\right)},
$$

where $N_{L R}\left(\omega_{\ell}\right):=n_{L}\left(\omega_{\ell}\right)+n_{R}\left(\omega_{\ell}\right)$ for $\rho_{(12)}$ and $\rho_{(23)}$, while

$$
c=\frac{N_{L R}\left(\omega_{1}\right)+N_{L R}\left(\omega_{3}\right)-2 N_{L R}\left(\omega_{2}\right)}{8\left(1+N_{L R}\left(\omega_{1}\right)\right)\left(1+N_{L R}\left(\omega_{2}\right)\left(1+N_{L R}\left(\omega_{3}\right)\right)\right.}
$$

for $\rho_{(13)}$. Unlike for the spin [Eqs. (75) and (76)] and heat [Eq. (81)] flows, which depend on the differences $n_{L}\left(\omega_{\ell}\right)-$ $n_{R}\left(\omega_{\ell}\right), c$ need not vanish even for identical baths, so 
that $n_{L}\left(\omega_{\ell}\right)=n_{R}\left(\omega_{\ell}\right)=n\left(\omega_{\ell}\right)$ for $\ell=1,2,3$. Indeed, in the latter case the stationary state is the Gibbs thermal state given by Eq. (62), which can carry two-spin quantum correlations because of the interspin interactions.

\section{Two-spin concurrence in an $N$-spin chain}

In order to compute the concurrence in the general case of an $N$-spin chain, one needs the coefficients in Eqs. (88)-(90); for that purpose, one has to select from each $p \times p$ matrix $\mathcal{S}^{(p)}$ the entries specified by the indices $\mathcal{N}_{p}^{(r s)}(0,0), \mathcal{N}_{p}^{(r s)}(0,1), \mathcal{N}_{p}^{(r s)}(1,0)$, and $\mathcal{N}_{p}^{(r s)}(1,1)$. These combinadic indices correspond to a total number of 1 's equal to $p$ : among them, the indices $\mathcal{N}_{p}^{(r s)}(0,0)$ number binary strings with zeroes at sites $r$ and $s$ and $p$ 1's over the remaining $N-2$ sites, $\mathcal{N}_{p}^{(r s)}(0,1)$ and $\mathcal{N}_{p}^{(r s)}(1,0)$ those binary strings with 1 at sites $s$ and $r$, respectively $r$ and $p-11$ 's over the remaining $N-2$ sites and, finally, the combinadic indices $\mathcal{N}_{p}^{(r s)}(1,1)$ list the binary strings with two 1's at sites $r$ and $s$ and $p-21$ 's distributed over the remaining $N-2$ sites. We reconstruct such combinadic indices by means of the choices $i_{1}<\cdots i_{p}, i_{1}<\cdots<$ $i_{p-1}$, and $i_{1}<\cdots<i_{p-2}$ among the $N-2$ sites at which the 1's not already allocated at $r$ and/or $s$ can be assigned.

In order to do this, let us first consider $\mathcal{N}_{p}^{(r s)}(0,0)$ : according to Eq. (82): $\mathcal{N}_{p}^{(r s)}(0,0)$ labels all $N$-tuple with $p$ 1's distributed over all sites except the site $r$ and the site $s$. There are at most $\left(\begin{array}{c}N-2 \\ p\end{array}\right)$ such sites if $0 \leq p \leq N-2$; let $i_{1}<\cdots<i_{p}$ be the sites with $i_{\ell} \neq r, s$ chosen among

$$
1,2, \ldots, r-1, r+1, \ldots, s-1, s+1, \ldots, N
$$

Then, the required indices are of the form

$$
\mathcal{N}_{p}^{(r s)}(0,0)=1+\sum_{\ell=1}^{p}\left(\begin{array}{c}
i_{\ell}-1 \\
\ell
\end{array}\right) .
$$

In the case of the combinadic indices $\mathcal{N}_{p}^{(r s)}(0,1)$, there are $p-11$ 's to be distributed over the $N-2$ sites in Eq. (K42) and $\left(\begin{array}{c}N-2 \\ p-1\end{array}\right)$ such choices, the binomial vanishing if $p<N-2 . \mathcal{N}_{p}^{(r s)}(0,1)$ signals a 1 at site $s$.

Let $i_{1}<\cdots<i_{p-1}$ be the positions of the other 1's and let $i_{s^{*}}$ denote the largest $0 \leq i_{\ell}<s$, corresponding to the following distribution of 1 's:

$$
0<i_{1}<\cdots<i_{s^{*}}<s<i_{s^{*}+1}<\cdots<i_{p-1} .
$$

Then, with the proviso that $s^{*}=0$, if $i_{s *}=0$, that is, when all other 1's occurs at sites beyond $s$, and that the sums are set to zero if the first summation index is smaller than the last one, the required combinadic indices are retrieved as

$$
\begin{aligned}
\mathcal{N}_{p}^{(r s)}(0,1)= & 1+\sum_{\ell=1}^{s^{*}}\left(\begin{array}{c}
i_{\ell}-1 \\
\ell
\end{array}\right)+\left(\begin{array}{c}
s-1 \\
s^{*}+1
\end{array}\right) \\
& +\sum_{\ell=s^{*}+1}^{p-1}\left(\begin{array}{c}
i_{\ell}-1 \\
\ell+1
\end{array}\right) .
\end{aligned}
$$

Analogously, when a 1 occurs at site $r$, then

$$
\begin{aligned}
\mathcal{N}_{p}^{(r s)}(1,0)= & 1+\sum_{\ell=1}^{r^{*}}\left(\begin{array}{c}
i_{\ell}-1 \\
\ell
\end{array}\right)+\left(\begin{array}{c}
r-1 \\
r^{*}+1
\end{array}\right) \\
& +\sum_{\ell=r^{*}+1}^{p-1}\left(\begin{array}{c}
i_{\ell}-1 \\
\ell+1
\end{array}\right) .
\end{aligned}
$$

Finally, in the case of two 1's at $r$ and $s$, there remain another $p-21$ 's to be distributed among the $N-2$ sites in Eq. (K42), thus making for $\left(\begin{array}{c}N-2 \\ p-2\end{array}\right)$ indices $\mathcal{N}_{p}^{(r s)}(1,1)$. Then, the combinadic index

$$
\begin{aligned}
\mathcal{N}_{p}^{(r s)}(1,1)= & 1+\sum_{\ell=1}^{r^{*}}\left(\begin{array}{c}
i_{\ell}-1 \\
\ell
\end{array}\right)+\left(\begin{array}{c}
r-1 \\
r^{*}+1
\end{array}\right) \\
& +\sum_{\ell=r^{*}+1}^{s^{*}}\left(\begin{array}{c}
i_{\ell}-1 \\
\ell+1
\end{array}\right)+\left(\begin{array}{c}
s-1 \\
s^{*}+2
\end{array}\right) \\
& +\sum_{\ell=s^{*}+1}^{p-2}\left(\begin{array}{c}
i_{\ell}-1 \\
\ell+2
\end{array}\right) .
\end{aligned}
$$

corresponds to a choice of $p-21$ 's of the form

$$
\begin{aligned}
i_{1} & <\cdots<i_{r^{*}}<r<i_{r^{*}+1} \\
& <\cdots<\cdots<i_{s^{*}}<s<i_{s^{*}+1}<\cdots<i_{p-2}
\end{aligned}
$$

with the positions $i_{1}<\cdots<i_{p-2}$ of the 1 's in an $N$-tuple where two 1's are already present at sites $r<s$ and $r^{*}$ and with $s^{*}$ denoting the largest integers such that $i_{r^{*}}<r$ and $i_{s^{*}}<s$.

Note that by setting $r^{*}=0$ and $s^{*}=0$ if $i_{r *}=0$ and $i_{s *}=0$ and with the convention about the sums introduced before Eq. (K44), the above expression also accounts for the cases when $i_{r^{*}}=i_{s^{*}}=0$, which corresponds to having all 1 's at sites beyond $s$,

$$
0<r<s<i_{1}<\cdots<i_{p-2},
$$

the cases when there are 1's before $r$, but no 1's in between $r$ and $s$, that is, when $0<i_{r^{*}}, i_{s *}=0$,

$$
0<i_{1}<\cdots<i_{r^{*}}<r<s<i_{r^{*}+1}<\cdots<i_{p-2}
$$


and the cases when there are no 1's before $r$, but $s^{*} 1$ 's before $s$, that is, when $i_{r^{*}}=0$ and $i_{s^{*}}>0$,

$$
0<r<i_{1}<\cdots<i_{s^{*}}<s<i_{s^{*}+1}<\cdots<i_{p-2} .
$$

[1] S. Datta, Quantum Transport: Atom to Transistor (Cambridge University Press, Cambridge, 2005).

[2] J. Gemmer, M. Michel, and G. Mahler, Quantum Thermodynamics, Lect. Notes Phys. Vol. 784 (Springer-Verlag, Berlin, 2009).

[3] V. May and O. Kühn, Charge and Energy Transfer Dynamics in Molecular Systems (Wiley, Weinheim, 2011), 3rd ed.

[4] G. Benenti, G. Casati, K. Saito, and R. S. Whitney, Fundamental aspects of steady-state conversion of heat to work at the nanoscale, Phys. Rep. 694, 1 (2017).

[5] S. Lepri, R. Livi, and A. Politi, Thermal conduction in classical low-dimensional lattices, Phys. Rep. 377, 1 (2003).

[6] F. Caruso, A. W. Chin, A. Datta, S. F. Huelga, and M. B. Plenio, Highly efficient energy excitation transfer in light-harvesting complexes: The fundamental role of noise-assisted transport, J. Chem. Phys. 131, 105106 (2009).

[7] J. T. Barreiro, M. Müller, P. Schindler, D. Nigg, T. Monz, M. Chwalla, M. Hennrich, C. F. Roos, P. Zoller, and R. Blatt, An open-system quantum simulator with trapped ions, Nature 470, 486 (2011).

[8] J. Wu and M. Berciu, Heat transport in quantum spin chains: Relevance of integrability, Phys. Rev. B 83, 214416 (2011).

[9] F. Giazotto and M. J. Martinez-Perez, The Josephson heat interferometer, Nature 492, 401 (2012).

[10] J.-P. Brantut, C. Grenier, J. Meineke, D. Stadler, S. Krinner, C. Kollath, T. Esslinger, and A. Georges, A thermoelectric heat engine with ultracold atoms, Science 342, 713 (2013).

[11] J.-P. Brantut, C. Grenier, J. Meineke1, D. Stadler, S. Krinner, C. Kollath, T. Esslinger, and A. Georges, A Thermoelectric Heat Engine with Ultracold Atoms, Science 342, 713 (2013).

[12] R. Labouvie, B. Santra, S. Heun, and H. Ott, Bistability in a Driven-Dissipative Superfluid, Phys. Rev. Lett. 116, 235302 (2016).

[13] F. Schlawin, K. E. Dorfman, B. P. Fingerhut, and S. Mukamel, Suppression of population transport and control of exciton distributions by entangled photons, Nat. Commun. 4, 1782 (2013).

[14] A. Bermudez, M. Bruderer, and M. B. Plenio, Controlling and Measuring Quantum Transport of Heat in TrappedIon Crystals, Phys. Rev. Lett. 111, 040601 (2013).

[15] B. Leggio, R. Messina, and M. Antezza, Thermally activated nonlocal amplification in quantum energy transport, Europhys. Lett. 110, 40002 (2015).

[16] N. Freitas, E. A. Martinez, and J. P. Paz, Heat transport through ion crystals, Phys. Scr. 91, 013007 (2016).
[17] B. Dutta, J. T. Peltonen, D. S. Antonenko, M. Meschke, M. A. Skvortsov, B. Kubala, J. König, C. B. Winkelmann, H. Courtois, and J. P. Pekola, Thermal Conductance of a Single-Electron Transistor, Phys. Rev. Lett. 119, 077701 (2017).

[18] P. Doyeux, R. Messina, B. Leggio, and M. Antezza, Excitation injector in an atomic chain: Long-range transport and efficiency amplification, Phys. Rev. A 95, 012138 (2017).

[19] R. Biele, C. A. Rodríguez-Rosario, T. Frauenheim, and A. Rubio, Controlling heat and particle currents in nanodevices by quantum observation, npj Quantum Mater. 2, 38 (2017).

[20] B. Bertini, F. Heidrich-Meisner, C. Karrasch, T. Prosen, R. Steinigeweg, M. Žnidaric, Finite-temperature transport 1498 in one-dimensional quantum lattice models, Rev. Mod. Phys. 93, 025003 (2021).

[21] L.-A. Wu and D. Segal, Energy flux operator, current conservation and the formal Fourier's law, J. Phys. A 42, 025302 (2009).

[22] R. Alicki and K. Lendi, Quantum Dynamical Semigroups and Applications, Lect. Notes Phys. Vol. 717 (SpringerVerlag, Berlin, 2007).

[23] A. Rivas and S. F. Huelga, Open Quantum Systems (Springer-Verlag, Berlin, 2012).

[24] F. Benatti and R. Floreanini, Open quantum dynamics: Complete positivity and entanglement, Int. J. Mod. Phys. B 19, 3063 (2005).

[25] F. Benatti, Dynamics, Information and Complexity in Quantum Systems (Springer-Verlag, Berlin, 2009).

[26] R. Alicki, in: Lect. Notes Phys. Vol. 597, edited by P. Garbaczewski and R. Olkiewicz (Springer-Verlag, Berlin, 2002), p. 239.

[27] F. Benatti and R. Floreanini, Eds., Dissipative Quantum Dynamics, Lect. Notes Phys. Vol. 622 (Springer-Verlag, Berlin, 2003).

[28] A. Kossakowski, On necessary and sufficient conditions for a generator of a quantum dynamical semigroup, Bull. Acad. Pol. Sc., Sci. Ser. Math. Astro. Phys. 20, 1021 (1972).

[29] E. B. Davies, Markovian master equations, Commun. Math. Phys. 39, 91 (1974).

[30] E. B. Davies, Markovian master equations. II, Math. Ann. 219, 147 (1976).

[31] E. B. Davies, Quantum Theory of Open Systems (Academic Press, New York, 1976).

[32] V. Gorini, A. Kossakowski, and E. C. G. Sudarshan, Completely positive dynamical semigroups of $N$-level systems, J. Math. Phys. 17, 821 (1976).

[33] G. Lindblad, On the generators of quantum dynamical semigroups, Commun. Math. Phys. 48, 119 (1976).

[34] V. Gorini, A. Frigerio, M. Verri, A. Kossakowski, and E. G. C. Sudarshan, Properties of quantum Markovian master equations, Rep. Math. Phys. 13, 149 (1976).

[35] R. Dümcke and H. Spohn, The proper form of the generator in the weak coupling limit, Z. Phys. B 34, 419 (1979).

[36] H. Spohn, Kinetic equations from Hamiltonian dynamics: Markovian limits, Rev. Mod. Phys. 52, 569 (1980). 
[37] M. Merkli, Quantum Markovian master equations: Resonance theory shows validity for all time scales, Ann. Phys. 412, 167996 (2020).

[38] E. B. Davies, A model of heat conduction, J. Stat. Phys. 18, 161 (1978).

[39] R. Alicki, The quantum open system as a model of the heat engine, J. Phys. A: Math. Gen. 12, L103 (1979).

[40] H. Spohn and J. L. Lebowitz, Irreversible thermodynamics for quantum systems weakly coupled to thermal reservoirs, Adv. Chem. Phys. 38, 109 (1978).

[41] H. Zoubi, M. Orenstien, and A. Ron, Dissipations in coupled quantum systems, Phys. Rev. A 67, 063813 (2003).

[42] H. Wichterich, M. J. Henrich, H.-P. Breuer, J. Gemmer, and M. Michel, Modeling heat transport through completely positive maps, Phys. Rev. E 76, 031115 (2007).

[43] A. Rivas, A. D. Plato, S. F. Huelga, S. F. Huelga, and M. B. Plenio, Markovian master equations: A critical study, New J. Phys. 12, 113032 (2010).

[44] R. Migliore, M. Scala, A. Napoli, K. Yuasa, H. Nakazato, and A. Messina, Dissipative effects on a generation scheme of a W state in an array of coupled Josephson junctions, J. Phys. B 44, 075503 (2011).

[45] J. P. Santos and F. L. Semiao, Master equation for dissipative interacting qubits in a common environment, Phys. Rev. A 89, 022128 (2014).

[46] T. Werlang and D. Valente, Heat transport between two pure-dephasing reservoirs, Phys. Rev. E 91, 012143 (2015).

[47] J. P. Santos and G. T. Landi, Microscopic theory of a nonequilibrium open bosonic chain, Phys. Rev. E 94, 062143 (2016).

[48] A. Rivas and M. A. Martin-Delgado, Topological heat transport and symmetry-protected boson currents, Sci. Rep. 7, 6350 (2017).

[49] As finding eigenvalues and eigenvectors of the system Hamiltonian might in general be laboriously difficult, an alternative approach has been often advocated, consisting in neglecting the interspin interaction in the derivation of the master equation (see, e.g., Refs. [67-98]). Although the two approaches, named global and local, are regularly adopted in applications and compared [41,43-46,88], [60, 64-66,99-110], it turns out that the local approach might not be able to capture all the correct system transport properties [50].

[50] F. Benatti, R. Floreanini, and L. Memarzadeh, Bathassisted transport in a three-site spin chain: Global versus local approach, Phys. Rev. A 102, 042219 (2020).

[51] P. Coleman, Introduction to Many-Body Physics (Cambridge University Press, Cambridge, 2015).

[52] N. Wu, Determinant representations of spin-operator matrix elements in the $X X$ spin chain and their applications, Phys. Rev. B 97, 014301 (2018).

[53] H. Spohn, Approach to equilibrium for completely positive dynamical semigroups of $N$-level systems, Rep. Math. Phys. 10, 189 (1976).

[54] A. Frigerio, Quantum dynamical semigroups and approach to equilibrium, Lett. Math. Phys. 2, 79 (1977).

[55] A. Frigerio, Stationary states of quantum dynamical semigroups, Commun. Math. Phys. 63, 269 (1977).
[56] D. E. Evans, Irreducible quantum dynamical semigroups, Commun. Math. Phys. 54, 293 (1977).

[57] F. Fagnola and R. Rebolledo, On the existence of stationary states for quantum dynamical semigroups, J. Math. Phys. 42, 1296 (2001).

[58] F. Fagnola and R. Rebolledo, Algebraic conditions for convergence of a quantum Markov semigroup to a steady state, Infin. Dimens. Anal. Quantum 11, 467 (2008).

[59] R. Gebauer and R. Car, Current in Open Quantum Systems, Phys. Rev. Lett. 93, 160404 (2004).

[60] A. Levy and R. Kosloff, The local approach to quantum transport may violate the second law of thermodynamics, Europhys. Lett. 107, 20004 (2014).

[61] A. B. Siddique, S. Farid, and M. Tahir, Proof of bijection for combinatorial number system, ArXiv:1601.05794 (2016).

[62] S. Hill and W. K. Wootters, Entanglement of a Pair of Quantum Bits, Phys. Rev. Lett. 78, 5022 (1997).

[63] W. K. Wootters, Entanglement of Formation of an Arbitrary State of Two Qubits, Phys. Rev. Lett. 80, 2245 (1998).

[64] S. Khandelwal, N. Palazzo, N. Brunner, and G. Haack, Critical heat current for operating an entanglement engine, New J. Phys. 22, 073039 (2020).

[65] A. S. Trushechkin and I. V. Volovich, Perturbative treatment of inter-site couplings in the local description of open quantum networks, Europhys. Lett. 113, 30005 (2016).

[66] J. B. Brask, G. Haack, N. Brunner, and M. Huber, Autonomous quantum thermal machine for generating steady-state entanglement, New J. Phys. 17, 113029 (2015).

[67] M. Michel and O. Hess, Transport in open spin chains: A Monte Carlo wave-function approach, Phys. Rev. B 77, 104303 (2008).

[68] D. Karevski and T. Platini, Quantum Nonequilibrium Steady States Induced by Repeated Interactions, Phys. Rev. Lett. 102, 207207 (2009).

[69] M. Znidaric, Spin Transport in a One-Dimensional Anisotropic Heisenberg Model, Phys. Rev. Lett. 106, 220601 (2011).

[70] T. Prosen, Exact Nonequilibrium Steady State of a Strongly Driven Open $X X Z$ Chain, Phys. Rev. Lett. 107, 137201 (2011).

[71] T. Prosen and M. Znidaric, Diffusive high-temperature transport in the one-dimensional Hubbard model, Phys. Rev. B 86, 125118 (2012).

[72] T. Prosen, Comments on a boundary-driven open $X X Z$ chain: Asymmetric driving and uniqueness of steady states, Phys. Scr. 86, 058511 (2012).

[73] T. Prosen, Matrix product solutions of boundary driven quantum chains, J. Phys. A 48, 373001 (2015).

[74] V. Popkov, Alternation of sign of magnetization current in driven XXZ chains with twisted XY boundary gradients, J. Stat. Mech. 2012, P12015 (2012).

[75] J. J. Mendoza-Arenas, S. Al-Assam, S. R. Clark, and D. Jaksch, Heat transport in the XXZ spin chain: from ballistic to diffusive regimes and dephasing enhancement, J. Stat. Mech. 2013, P07007 (2013). 
[76] D. Karevski, V. Popkov, and G. M. Schütz, Exact Matrix Product Solution for the Boundary-Driven Lindblad $X X Z$ Chain, Phys. Rev. Lett. 110, 047201 (2013).

[77] L. A. Correa, J. P. Palao, G. Adesso, and D. Alonso, Performance bound for quantum absorption refrigerators, Phys. Rev. E 87, 042131 (2013).

[78] V. Popkov and M. Salerno, Anomalous currents in a driven XXZ chain with boundary twisting at weak coupling or weak driving, J. Stat. Mech. 2013, P02040 (2013).

[79] A. Asadian, D. Manzano, M. Tiersch, and H. J. Briegel, Heat transport through lattices of quantum harmonic oscillators in arbitrary dimensions, Phys. Rev. E 87, 012109 (2013).

[80] V. Popkov, M. Salerno, and R. Livi, Manipulating energy and spin currents in non-equilibrium systems of interacting qubits, New J. Phys. 15, 023030 (2013).

[81] G. T. Landi, E. Novais, M. J. de Oliveira, and D. Karevski, Flux rectification in the quantum $X X Z$ chain, Phys. Rev. E 90, 042142 (2014).

[82] D. Manzano and P. I. Hurtado, Symmetry and the thermodynamics of currents in open quantum systems, Phys. Rev. B 90, 125138 (2014).

[83] J. Cui, J. I. Cirac, and M. C. Banuls, Variational Matrix Product Operators for the Steady State of Dissipative Quantum Systems, Phys. Rev. Lett. 114, 220601 (2015).

[84] V. Popkov, M. Salerno, and R. Livi, Full decoherence induced by local fields in open spin chains with strong boundary couplings, New J. Phys. 17, 023066 (2015).

[85] F. Nicacio, A. Ferraro, A. Imparato, M. Paternostro, and F. L. Semiao, Thermal transport in out-of-equilibrium quantum harmonic chains, Phys. Rev. E 91, 042116 (2015).

[86] G. T. Landi and D. Karevski, Open Heisenberg chain under boundary fields: A magnonic logic gate, Phys. Rev. B 91, 174422 (2015).

[87] L. Schuab, E. Pereira, and G. T. Landi, Energy rectification in quantum graded spin chains: Analysis of the $X X Z$ model, Phys. Rev. E 94, 042122 (2016).

[88] P. H. Guimaraes, G. T. Landi, and M. J. de Oliveira, Nonequilibrium quantum chains under multisite Lindblad baths, Phys. Rev. E 94, 03213 (2016).

[89] S. Campbell, G. De Chiara, and M. Paternostro, Equilibration and nonclassicality of a double-well potential, Sci. Rep. 6, 19730 (2016).

[90] D. Manzano, C. Chuang, and J. Cao, Quantum transport in $d$-dimensional lattices, New J. Phys. 18, 043044 (2016).

[91] C. Monthus, Boundary-driven Lindblad dynamics of random quantum spin chains: Strong disorder approach for the relaxation, the steady state and the current, J. Stat. Mech. 2017, 043303 (2017).

[92] G. De Chiara, G. Landi, A. Hewgill, B. Reid, A. Ferraro, A. J. Roncaglia, and M. Antezza, Reconciliation of quantum local master equations with thermodynamics, New J. Phys. 20, 113024 (2018).

[93] F. Carollo, J. P. Garrahan, I. Lesanovsky, and C. PerezEspigares, Fluctuating hydrodynamics, current fluctuations, and hyperuniformity in boundary-driven open quantum chains, Phys. Rev. E 96, 052118 (2017).
[94] F. Carollo, J. P. Garrahan, and I. Lesanovsky, Current fluctuations in boundary-driven quantum spin chains, Phys. Rev. B 98, 094301 (2018).

[95] T. Chanda, T. Das, D. Sadhukhan, A. K. Pal, A. Sen, and U. Sen, Scale-invariant freezing of entanglement, Phys. Rev. A 97, 062324 (2018).

[96] E. Pereira, Heat, work, and energy currents in the boundary-driven $X X Z$ spin chain, Phys. Rev. E 97, 022115 (2018).

[97] M. Brenes, E. Mascarenhas, M. Rigol, and J. Goold, Hightemperature coherent transport in the $X X Z$ chain in the presence of an impurity, Phys. Rev. B 98, 235128 (2018).

[98] K. V. Hovhannisyan and A. Imparato, Quantum current in dissipative systems, New J. Phys. 21, 052001 (2019).

[99] G. L. Decordi and A. Vidiella-Barranco, Two coupled qubits interacting with a thermal bath: A comparative study of different models, Opt. Commun. 387, 366 (2017).

[100] J. T. Stockburger and T. Motz, Thermodynamic deficiencies of some simple Lindblad operators, Fortschr. Phys. 65, 1600067 (2017).

[101] J. O. Gonzalez, L. A. Correa, G. Nocerino, J. P. Palao, D. Alonso, and G. Adesso, Testing the validity of the "local" and "global" GKLS master equations on an exactly solvable model, Open Syst. Inf. Dyn. 24, 1740010 (2017).

[102] G. G. Giusteri, F. Recrosi, G. Schaller, and G. L. Celardo, Interplay of different environments in open quantum systems: Breakdown of the additive approximation, Phys. Rev. E 96, 012113 (2017).

[103] P. P. Hofer, M. Perarnau-Llobet, L. D. Miranda, G. Haack, R. Silva, J. B. Brask, and N. Brunner, Markovian master equations for quantum thermal machines: Local versus global approach, New J. Phys. 19, 123037 (2017).

[104] M. Tahir Naseem, A. Xuereb, and O. E. Mustecaplioglu, Thermodynamic consistency of the optomechanical master equation, Phys. Rev. A 98, 052123 (2018).

[105] N. Shammah, S. Ahmed, N. Lambert, S. De Liberato, and F. Nori, Open quantum systems with local and collective incoherent processes: Efficient numerical simulations using permutational invariance, Phys. Rev. A 98, 063815 (2018).

[106] J. Kolodynski, J. B. Brask, M. Perarnau-Llobet, and B. Bylicka, Adding dynamical generators in quantum master equations, Phys. Rev. A 97, 062124 (2018).

[107] M. T. Mitchison and M. Plenio, Non-additive dissipation in open quantum networks out of equilibrium, New J. Phys. 20, 033005 (2018).

[108] E. Mascarenhas, F. Damanet, S. Flannigan, L. Tagliacozzo, A. J. Daley, J. Goold, and I. De Vega, Nonreciprocal quantum transport at junctions of structured leads, Phys. Rev. B 99, 245134 (2019).

[109] M. Cattaneo, G. L. Giorgi, S. Maniscalco, and R. Zambrini, Local versus global master equation with common and separate baths: Superiority of the global approach in partial secular approximation, New J. Phys. 21, 113045 (2019).

[110] D. Farina, G. De Filippis, V. Cataudella, M. Polini, and V. Giovannetti, Going beyond local and global approaches for localized thermal dissipation, Phys. Rev. A 102, 052208 (2020). 